\title{
Laboratory and Flight Tests of 2D Imaging Probes: Toward a Better Understanding of Instrument Performance and the Impact on Archived Data
}

\author{
Colin Gurganus and Paul Lawson \\ SPEC Incorporated, Boulder, Colorado
}

(Manuscript received 23 November 2017, in final form 9 May 2018)

\begin{abstract}
Two-dimensional (2D) imaging probes, such as the $2 \mathrm{D}$ stereo (2D-S) probe and the cloud imaging probe (CIP), are routinely used to provide in situ measurements of cloud particle properties. The basic measurement is shadowgraphs of water drops and ice particles from which particle size distributions, projected particle area, and mass concentrations are determined. These data permeate data archives of domestic and foreign government agencies, universities, and the private sector. This paper provides results from laboratory tests and flight tests on a Learjet research aircraft that give new insights into the performance of the 2D imaging probes, and how their performance may have impacted measurements collected in data archives. The laboratory tests are conducted with the aid of two devices: 1) a droplet generator that provides known concentrations of water drops from 15 to $65 \mu \mathrm{m} \pm 1 \mu \mathrm{m}$ that can be positioned in the probe's sample volume with 10 - $\mu \mathrm{m}$ precision; and 2) a motorized spinning platform that supports transparent disks with small opaque features (i.e., a "spinning disk"), which replicates the effect of particles transecting the probe's sample volume at translational speeds up to $190 \mathrm{~m} \mathrm{~s}^{-1}$. The flight tests were conducted with a Learjet research aircraft that collected cloud particle data at true airspeeds from 99 to $170 \mathrm{~m} \mathrm{~s}^{-1}$. The results provide new insights into how probe optics, time response, and data throughput of the $2 \mathrm{D}-\mathrm{S}$ and CIP electro-optics impact the measurements of cloud particles. The results, summarized in the conclusions, suggest how archived data are impacted.
\end{abstract}

\section{Introduction}

The advent of Particle Measuring Systems, Inc. (PMS) two-dimensional (2D) imaging probes (Knollenberg 1970) led to a quantum advance in cloud microphysical in situ measurement technology. For about 25 years, PMS populated 2D probes throughout the world until the company discontinued its manufacturing division. Droplet Measurement Technologies, Inc. (DMT) took the baton, upgraded the electronics in the 1990s and started manufacturing versions of the $2 \mathrm{D}$ probes called the cloud imaging probe (CIP) and the precipitation imaging probe (PIP) (Baumgardner et al. 2001). A decade later SPEC Inc. redesigned the optics and electronics of 2D imaging probes, developing the high volume precipitation spectrometer (HVPS) and 2D stereo (2D-S) cloud probe (Lawson et al. 2006). The 2D-S consists of two completely independent 2D imaging probes within one housing; the 2D-128 used in this study is a single-probe (often called single channel) version of the $2 \mathrm{D}-\mathrm{S}$.

Corresponding author: Dr. Paul Lawson, plawson@specinc.com
Two-dimensional image data are essential to several disciplines in the atmospheric sciences, and only a few of the more prominent examples are listed here. Basic cloud microphysics is focused on the conversion of cloud droplets into raindrops via coalescence and ice via freezing with subsequent growth via vapor deposition, aggregation, and riming (e.g., Lawson et al. 2015, 2017). The fundamental measurement of 2D imaging probes is particle projected area, which is a direct estimate of the particle cross-sectional area. Radiative transfer theory in clouds incorporates the image cross-sectional size distribution of particles (e.g., Jensen et al. 2009; Lawson et al. 2010). In situ 2D imaging measurements of precipitation provide verification for ground and satellite radar retrievals (e.g., Mitchell et al. 2010; Mace and Benson 2017). Two-dimensional imaging data are also used to estimate liquid and ice water contents, and while these are not a fundamental measurement of the probes, bulk properties can be readily derived from $2 \mathrm{D}$ images. More recently, measurements in the tropical tropopause layer (TTL) are of interest because of the effect that high-level cirrus can have on stratospheric water vapor (e.g., Solomon et al. 2010). Two-dimensional particle 
size measurements are used to estimate particle mass and number concentrations, leading to implications of homogeneous and heterogeneous nucleation mechanisms (Jensen et al. 2016).

A considerable amount of data has been collected using the PMS, DMT, and SPEC 2D probes installed on various research aircraft that have participated in dozens of major field experiments. Most of these measurements are archived in various databases maintained by domestic and international government agencies. Unfortunately, while government funding has been available for collecting data from the $2 \mathrm{D}$ imaging probes, there has been precious little funding available to rigorously quantify the performance of these probes. As a result, significant measurement uncertainties exist and remain poorly quantified, and researchers that access the archives are often uninformed of measurement uncertainties.

Albeit, several researchers have made sincere efforts to quantify the performance of $2 \mathrm{D}$ imaging probes, and these papers have improved our understanding of measurement uncertainties (e.g., Heymsfield and Parrish 1986; Korolev et al. 1991, 1998; Strapp et al. 2001; Baumgardner and Korolev 1997; Lawson et al. 2006; Wu and McFarquhar 2016; Hayman et al. 2016). Over the past decade, a calibration laboratory has slowly evolved at SPEC and the results have reached a level of maturity that are worthy of reporting in the open literature. The primary challenges were to 1 ) develop a high-speed (up to $190 \mathrm{~m} \mathrm{~s}^{-1}$ ) spinning disk with opaque images that can be used to quantify the time response of the complete electro-optics of the probe, and 2) develop a drop generator capable of generating droplets from about 10- to $70-\mu \mathrm{m}$ diameter with approximately $1-\mu \mathrm{m}$ accuracy, which can be positioned through the probe sample area to within an accuracy of $1 \mu \mathrm{m}$. Both of these calibration tools will be described in more detail in section 2 .

The purpose of this paper is to add to the atmospheric community's knowledge of the performance of $2 \mathrm{D}$ imaging probes and to provide some insight into how this performance affects data that are currently stored in archives. The takeaway message is that there are cloud conditions that produce uncertainties in some $2 \mathrm{D}$ probe measurements, and that the particle sizes and concentrations that induce these uncertainties can be estimated based on laboratory and flight data presented in this paper. This information will help researchers qualify their interpretation of $2 \mathrm{D}$ probe data and hopefully avoid drawing conclusions based on measurements with known uncertainties. We do not present "corrections" to archived data in this paper. Such corrections, if possible, will require more extensive laboratory and flight tests, and better forward models of probe performance.
We focus on the DMT CIP and the SPEC 2D-S probe, which have been responsible for the large majority of $2 \mathrm{D}$ image data collected in this century. The layout of this paper is as follows: Section 2 describes the calibration facility. Section 3 describes the 2D imaging probes. Sections 4 and 5 present the results from experiments in the calibration laboratory and flight tests with the Learjet research aircraft, respectively. Section 6 is a discussion and summary, and section 7 concludes the paper.

\section{Calibration facility}

The principal components of the calibration laboratory are the drop generator and the high-speed spinning disk, shown in the photographs in Fig. 1. The drop generator is based on the design described in Lance et al. (2010). Basically, droplets are generated using a commercial piezoelectric drop generator. Drop sizing is accomplished using two independent techniques: A laser beam is strobed at a frequency synchronized to the piezoelectric actuator signal with a variable delay control and drops are imaged with $0.6-\mu \mathrm{m}$ pixel resolution on a digital camera. For independent verification of a droplet's diameter, we also use the "glares technique" described in Korolev et al. (1991). More detail describing the drop generator and its applications are found in the appendix to this paper.

Knollenberg (1970) showed that the depth of field (DOF) follows the relationship:

$$
\mathrm{DOF}= \pm c r^{2} / \lambda
$$

where $r$ is the drop radius, $\lambda$ is the wavelength of (laser) light, and $c$ is a "constant" that can vary with different optical configurations. A maximum value of $c$ can be determined from Fresnel diffraction theory (Korolev et al. 1998). It is best to determine the value of $c$ for each optical system experimentally. The droplet generator was used to determine the value of $c$ for the 2D-128 probe. Droplets ranging from 29 to $67 \mu \mathrm{m}$ in diameter were precisely projected in $0.5-\mathrm{mm}$ steps across the DOF using the droplet generator. Figure 2 shows a plot of DOF versus $r^{2} / \lambda$ for both laboratory measurements and Zemax ray-tracing software, where $c$ is the value of slope of the best fit to the data. The laboratory measurements and Zemax simulation show very good agreement, resulting in a value of $c=8 \pm 0.2$. This also agrees with the value of $c=8.18$, derived by Korolev et al. (1998) from Fresnel diffraction theory. Additional examples and a more detailed description of how DOF is determined are found in the appendix to this paper.

The value of $c$ for the CIP could not be determined via Zemax because of the proprietary optical system. The manufacturer's CIP operator manual specifies a value of 


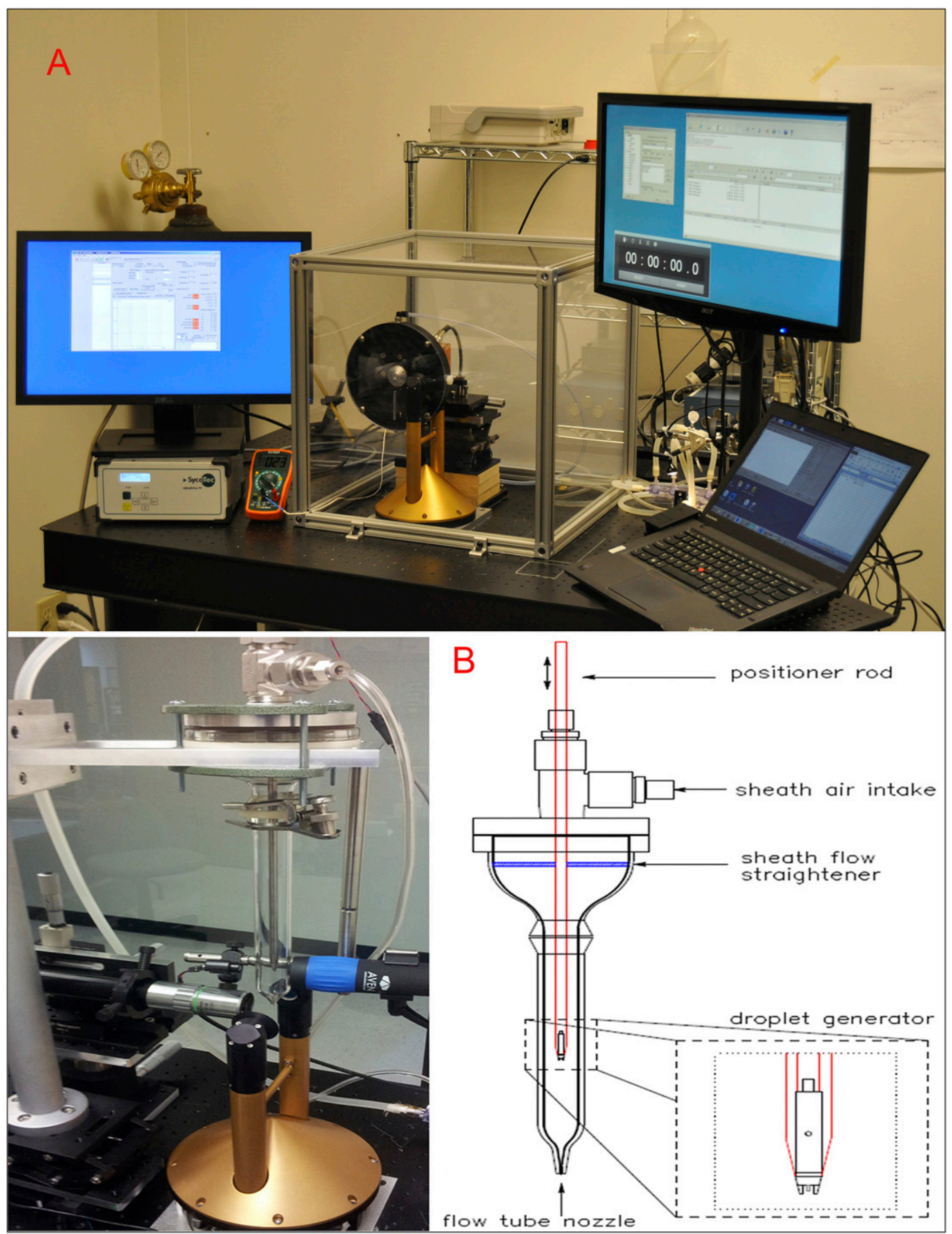

FIG. 1. Photographs of (a) high-speed spinning disk and (b) droplet generator with schematic adapted from Lance et al. (2010).

$c=3$, which is also the value given by Knollenberg (1970). For comparable droplet sizes $(D \sim 40 \mu \mathrm{m})$, it was observed that $c \geq 3$ for the two CIP instruments tested (see Fig. A2 in the appendix). For example, the CIP manufactured in 2017 and tested here indicates a much higher DOF range, which may result from changes to the imaging system (M. Freer 2018, personal communication). The drop generator cannot reliably make drops much larger than $65 \mu \mathrm{m}$ in diameter, so measurements over several CIP $25-\mu \mathrm{m}$ pixels is not possible

The high-speed spinning disk system is used to test the complete time response of the electro-optics of the 


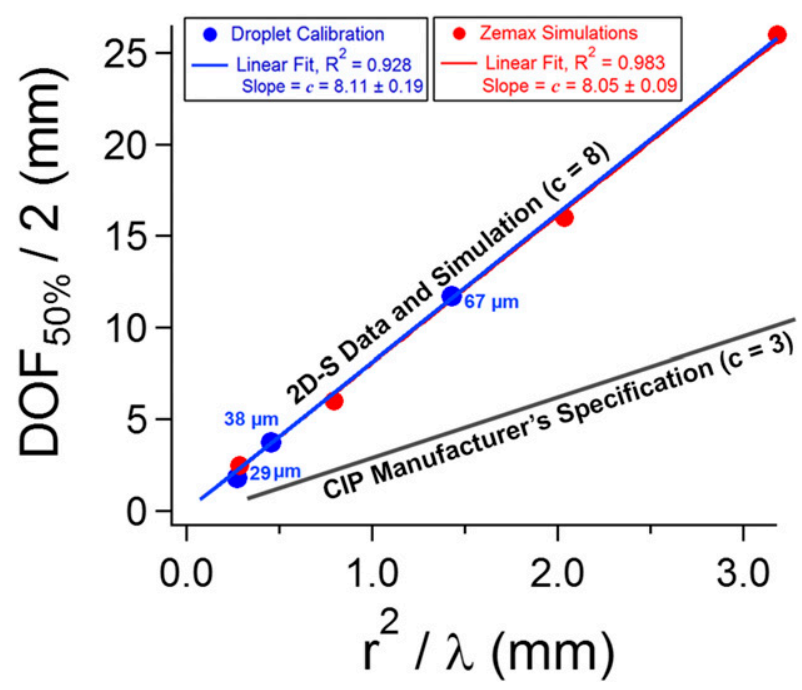

FIG. 2. Plot of 2D imaging probe DOF/2 vs $r^{2} / \lambda$ used to determine the value of $c$ in Eq. (1). Zemax ray-tracing software and data from the droplet generator shown for the 2D-S, and value of $c$ from manufacturer's manual and best fit to droplet data shown for CIP17.

imaging probes. Previous work has focused on the response time of the photodiode array and front-end amplifier (e.g., Baumgardner and Korolev 1997; Strapp et al. 2001; Hayman et al. 2016). However, there are other factors downstream of the front-end electronics that can also influence the total throughput of the electro-optics (see section 3). To simulate the effect of particles passing through the probe sample volume at aircraft speeds, disks with opaque dots and other features are spun at rotation speeds of up to $30000 \mathrm{rpm}$ $\left(190 \mathrm{~m}^{-1}\right.$ tangential velocity). An aluminum enclosure protects the experimenter in the event of high-speed mechanical failure, and a polycarbonate housing keeps dust and debris away from the disk (Fig. 1).

\section{2D imaging probes}

The CIP and 2D-S operate on the same principle: A collimated laser beam illuminates a linear array of positive-intrinsic-negative (PIN) photodiodes. The output of each photodiode in the array is measured at a rate proportional to the airspeed of the aircraft. When a particle passes over the array and occults some or all of the photodiodes, the probe electronics determines which photodiodes are "shadowed" by the particle and records a two-dimensional shadow image of the particle. While both the CIP and the 2D-S probe employ an identical principle of operation, they have different optics and electronics. It is beyond the scope of this paper to present a detailed comparison of the electro-optics in each probe. Instead, we examine the throughput of each probe using the calibration tools described in section 2 and flight tests with an instrumented research aircraft. When appropriate, we discuss some aspects of the electro-optics to help elucidate the results.

One difference in the designs of the CIP and 2D-S is that the CIP uses a 64-photodiode array, while the 2D-S employs a 128-photodiode array. A second difference is that the optics in the CIP are configured to make each pixel nominally equivalent to $25 \mu \mathrm{m}$ in the sample volume, while the $2 \mathrm{D}-\mathrm{S}$ optics are configured to generate nominally $10-\mu \mathrm{m}$ pixels in the sample volume. Other differences exist, and some are significant, but these features are intrinsic to the individual optical and electronic systems. Instead of attempting to identify and describe all of the intrinsic differences, here we evaluate the throughput of the entire electro-optics of each probe. This is accomplished using two calibration devices and the research aircraft. A precision droplet generator is used to define the optical depth of field of each probe. A high-speed spinning disk and the research aircraft are used to evaluate the complete throughput of the electrooptics as a function of relative particle speed. ${ }^{1}$

Figure 3 shows a block diagram of the fundamental components of the electro-optics of both the CIP and the 2D-S. Both the CIP and the 2D-S convert current signals from each photodiode to voltage using individual transimpedance amplifiers (TIA). The time response of a TIA is primarily a function of the amplifier specifications (gain, bandwidth, slew rate, etc.), feedback capacitorresistor time constant, and the capacitance of the photodiode. The output from each TIA passes through one or more additional amplifier stages to boost the signal voltage. The signal is then compared against an averaged threshold voltage and if the input signal voltage decreases below a preset level, the comparator output switches state, indicating that the photodiode is shadowed. A shadowed (occulted) photodiode is interpreted as a black pixel in the shadowgraph of a particle. Analog signals that originated at the output of the photodiode array are converted to digital signals by the comparators. From this point onward, the particle images are handled digitally as the data travels through buffers, data compression (in the case of the 2D-S) and transmission, and eventually to the data system for display and recording.

Cloud particles that pass through the sample area of 2D imaging probes at aircraft speeds can create very high data rates that exceed the throughput capability of

\footnotetext{
${ }^{1}$ Relative speed refers to the speed of a particle transecting the photodiode array. In this sense disk speed is analogous to airspeed.
} 


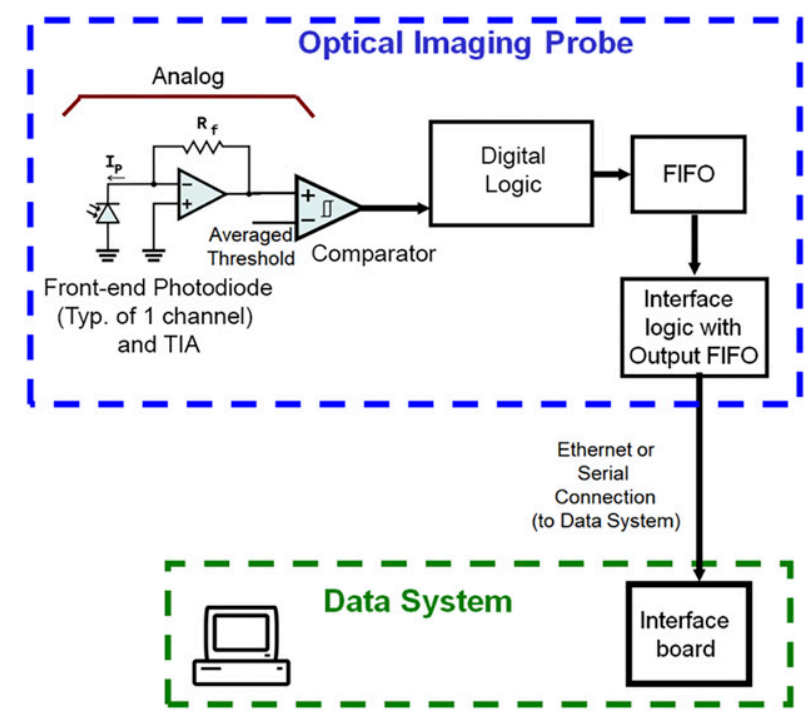

FIG. 3. The basic components and data flow of a 2D imaging probe.

the digital electronics. When this occurs the probe stops accumulating data and goes into a condition commonly called "overload," creating "dead time," a period of time when data are not being recorded. The 2D-S probe and the CIP handle overloads and dead time differently. The 2D-S electronics support a 16383 deep $\times$ 32-bitwide buffer that records the particle binary data, including the arrival time and transit time of each particle that is imaged. Additionally, the electronics produce a "flag" that is recorded in the data specifying the time the probe went into overload and when it again becomes active. All of this information-that is, particle arrival time, transit time, and overload flag-is recorded in the data stream to within one true airspeed clock period (i.e., $50 \mathrm{~ns}$ at $200 \mathrm{~m} \mathrm{~s}^{-1}$ ). Thus, when the $2 \mathrm{D}-\mathrm{S}$ goes into overload it records all of the dead time to within one clock period so that the sample volume can be adjusted accurately. Since each particle is precisely time stamped, shattered particles can be removed using particle interarrival times, and stuck bits and electronic noise can be removed using appropriate algorithms [see the appendix in Lawson (2011) for details].

The CIP records image data and estimates dead time based on the number of missed images. This is different from the technique used in the 2D-S and 2D-128, which measure dead time to within $50 \mathrm{~ns}$. CIP data are recorded in double fixed-length 4096-byte buffers. A time stamp is applied to the beginning and end of each buffer, as well as each particle within the buffer. However, there is no flag generated by the CIP when it goes into overload. When the CIP goes into overload, images are not recorded, but the electronics still process particle data, as described in the following paragraph.
A 16-bit particle counter is incremented whenever at least one photodiode is occulted. Following each particle image, the value of the particle counter is encoded as a 16-bit integer into a 64-bit word ("counter word"), along with a 64-bit word that records the time stamp ("timer word") are written in the buffer. If there is no dead time, then the counter word will increment by one bit for each image. If the counter word is incremented by more than one bit, then the number of missing bits can be used to count the number of missed particles during the time the probe was dead within a 4096-byte buffer (McFarquhar et al. 2017). This method differs from the $2 \mathrm{D}-\mathrm{S}$ in that the $2 \mathrm{D}-\mathrm{S}$ records the amount of time the probe was dead, while the CIP records the number of particles that are missed while the probe is dead. Each method can be employed to adjust the effective sample volume used to determine particle concentration when the probe is dead. However, by recording actual dead time, the $2 \mathrm{D}-\mathrm{S}$ accurately adjusts sample volume over the time period when the probe was active. On the other hand, recording the number of missed particles requires that certain conditions be met for the sample volume to be accurately adjusted.

For example, dead time occurring within a CIP buffer can be approximated to adjust the sample volume using an equation of the form (A. Korolev 2018, personal communication)

$$
\Delta t_{\text {dead }}=\Delta t\left(1-N_{\text {image }} / N_{\text {all }}\right),
$$

where $\Delta t_{\text {dead }}$ is the estimated dead time (s), $\Delta t$ is time period over which the buffer is recorded (s), $N_{\text {image }}$ is the number of images recorded by the $2 \mathrm{D}$ component of the probe, and $N_{\text {all }}$ is the number of particles counted by the $1 \mathrm{D}$ component of the probe. The sample volume is effectively adjusted using the ratio $N_{\text {image }} / N_{\text {all }}$. This adjustment can be used to estimate dead time if (at least) the following three conditions are met:

1) The terms $N_{\text {image }}$ and $N_{\text {all }}$ are large, that is, good counting statistics.

2) The particle concentration within the buffer is homogenous. If, for example, while the probe was dead it encountered a region with high droplet concentration and then encountered a region with a few large particles, or no particles at all, the correction to sample volume will not work well. As explained later, this scenario could be encountered in a mixed-phase cumulus updraft, or in anvil cirrus.

3) There have been no shattered particles or noise generated during dead time.

It is beyond the scope of this paper to quantify the uncertainty in particle size distributions resulting from 
estimates of CIP dead time, as this requires a representative forward model of the probe and simulations with several examples of particle size distributions. However, an estimate of hypothetical cloud conditions that will produce dead time in a CIP is worth considering. The manufacturer and independent assessments (A. Korolev 2018, personal communication) have determined that the CIP goes into overload and accumulates dead time when the data rate exceeds about 100 (4096 byte) buffers per second. Assume a monodisperse distribution of $100-\mu \mathrm{m}$-diameter drops (i.e., six 64-bit slices per image: four image slices, a timer word, and a counter word) passing through the probe at an airspeed of $150 \mathrm{~m} \mathrm{~s}^{-1}$. If we assume that all of the drops produce a $100-\mu \mathrm{m}$ image, then the CIP will go into overload, start producing dead time at a concentration of about $350 \mathrm{~L}^{-1}$, which equates to liquid water content of about $0.2 \mathrm{~g} \mathrm{~m}^{-3}$.

The CIP electronics also process data like a "1D probe" (Jensen and Granek 2002). A 1D probe estimates the maximum dimension of a particle by recording the maximum distance between any two occulted photodiodes. The data are sized and placed into size bins by the probe electronics. One-dimensional probe data cannot be corrected for several errors, for example, shattered particles, stuck bits, noise, and coincidence of two small particles that are interpreted as one large particle. Results from laboratory tests in the next section (see Table 2) show that as the data rate increases, the 1D particle count does not increase proportionally and will eventually plateau. This implies that the $1 \mathrm{D}$ component of the CIP is missing particles at the higher disk speeds. The manufacturer offers this feature as a convenience for real-time display and ready access to particle size concentrations, and not for quantitative assessment of the data (D. Baumgardner 2018, personal communication).

\section{Results of laboratory tests}

The laboratory tests were performed on a 2D-128, which is a "single channel" $2 \mathrm{D}-\mathrm{S}$ probe. Normally, the 2D-S probe has four arms with crossed laser beams in the sample area, effectively providing two independent measurements. To introduce a spinning disk in the sample area, one set of arms is removed, rendering a single-channel probe. Photographs of the 2D-128 and 2D-S are shown in Fig. 4. Two versions of the CIP are evaluated. One probe was purchased in 2002 (CIP02), and the second probe was purchased in 2017 (CIP17) and is incorporated into a combination cloud probe (CCP). Photographs of both the CIP and CCP are also shown in Fig. 4. The electronics and optics in the CIP have undergone modifications since its origin, and it is important to note that CIP17 performs much differently than CIP02. The only significant modification to the 2D-S electronics since its original manufacture has been to increase the clock and digital signal processor speed from 17 to $20 \mathrm{MHz}$. The 2D-128 probe in this study was manufactured in 2007 and is operating at $17 \mathrm{MHz}$.

Figure 5 shows close-up photographs of a transparent Mylar disk (in a black aluminum housing) and an aluminum disk with small quartz windows. After investigating several methodologies (printing, laser etching, lithography, etc.), we determined that it was impractical to manufacture and maintain a transparent disk without the very small $(<25 \mu \mathrm{m})$ surface defects that can withstand spinning at speeds near $200 \mathrm{~m} \mathrm{~s}^{-1}$. The size range of the defects, which are permanent, is from about 10 to $100 \mu \mathrm{m}$, with about $90 \% \leq 50 \mu \mathrm{m}$. A series of dots from 30 to $2000 \mu \mathrm{m}$, along with 250 and $500 \mu \mathrm{m}$ rosettes and a $200-\mu \mathrm{m}$ hexagon, are etched onto the Mylar disk (Fig. 6). The Fig. 6 caption provides more detail, describing each of the spinning disks. Size distributions of the small defects on the spinning disks are presented and discussed later in this section.

We were, however, able to manufacture an aluminum disk with small (5-mm diameter) clear sapphire windows without surface defects. A series of dots ranging from 11.4 to $120 \mu \mathrm{m}$ are lithographed onto the windows of the aluminum disk. As illustrated in Fig. 5, the 2D-128 probe successfully imaged a $11.4-\mu \mathrm{m}$ dot at $181 \mathrm{~m} \mathrm{~s}^{-1}$, which confirms the ability of the 2D-128 to image one-pixel particles at jet aircraft speeds. Unfortunately, the aluminum disk was not usable with the CIP. The CIP baseline restoration circuit averages the clear (unocculted) and opaque (occulted) portions of the disk. The opaque (aluminum) part of the disk occupies about $90 \%$ of the sample area, so the baseline never restores to the unocculted light level. This is generally not a problem in clouds where particles typically occupy $<5 \%$ of the average unocculted light. In contrast, the $2 \mathrm{D}-\mathrm{S}$ has a fast reset circuit. As a workaround, we have resorted to using a clear Mylar disk with images (dots, hexagons, and rosettes) printed on it and a clear acrylic disk with small irregular-shaped features.

A thin Mylar disk with dots and other geometric features was spun at speeds up to $190 \mathrm{~m} \mathrm{~s}^{-1}$. However, this disk tended to wobble slightly at higher disk speeds so that the smallest features could go in and out of focus, depending on small changes in disk speed. To prevent wobble, a thicker, more rigid acrylic disk that contained only small surface defects was also used. Photographs of the rigid disk under magnification showed that it did not wobble at any speed. The maximum speed of the rigid disks is $176 \mathrm{~m} \mathrm{~s}^{-1}$, because of its increased mass. 


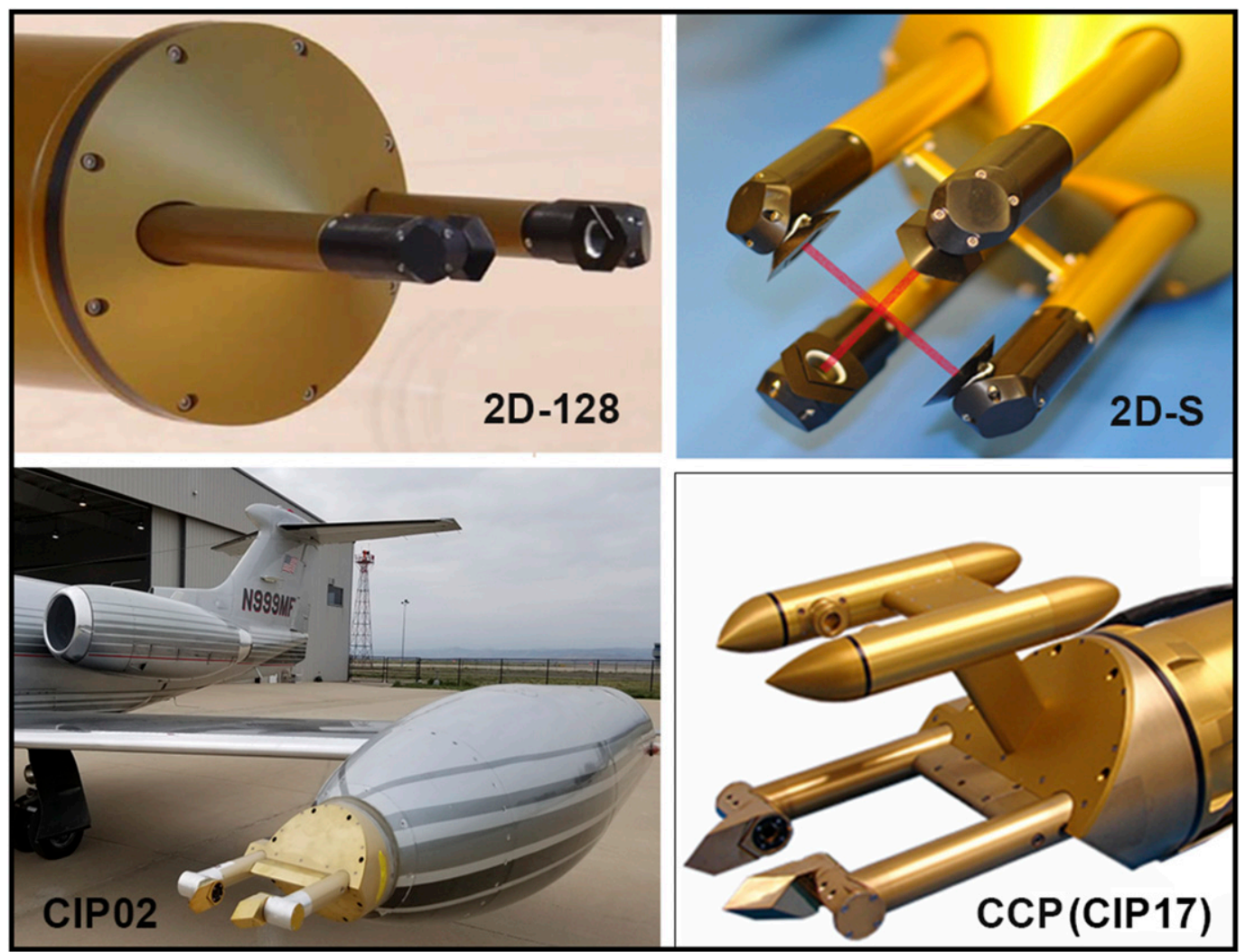

FIG. 4. Photographs of the 2D imaging probes discussed in this research.

Photographs of all three spinning disks (i.e., aluminum, Mylar, and acrylic) are shown in Fig. 6 along with a schematic showing the layout of dots, rosettes, and a hexagon that are printed on the Mylar disk.
Table 1 shows the size of each printed image on the Mylar disk and the number of pixels perpendicular to the disk rotation as a function of disk speed for both the 2D-128 and CIP02. The data in Table 1 show that the
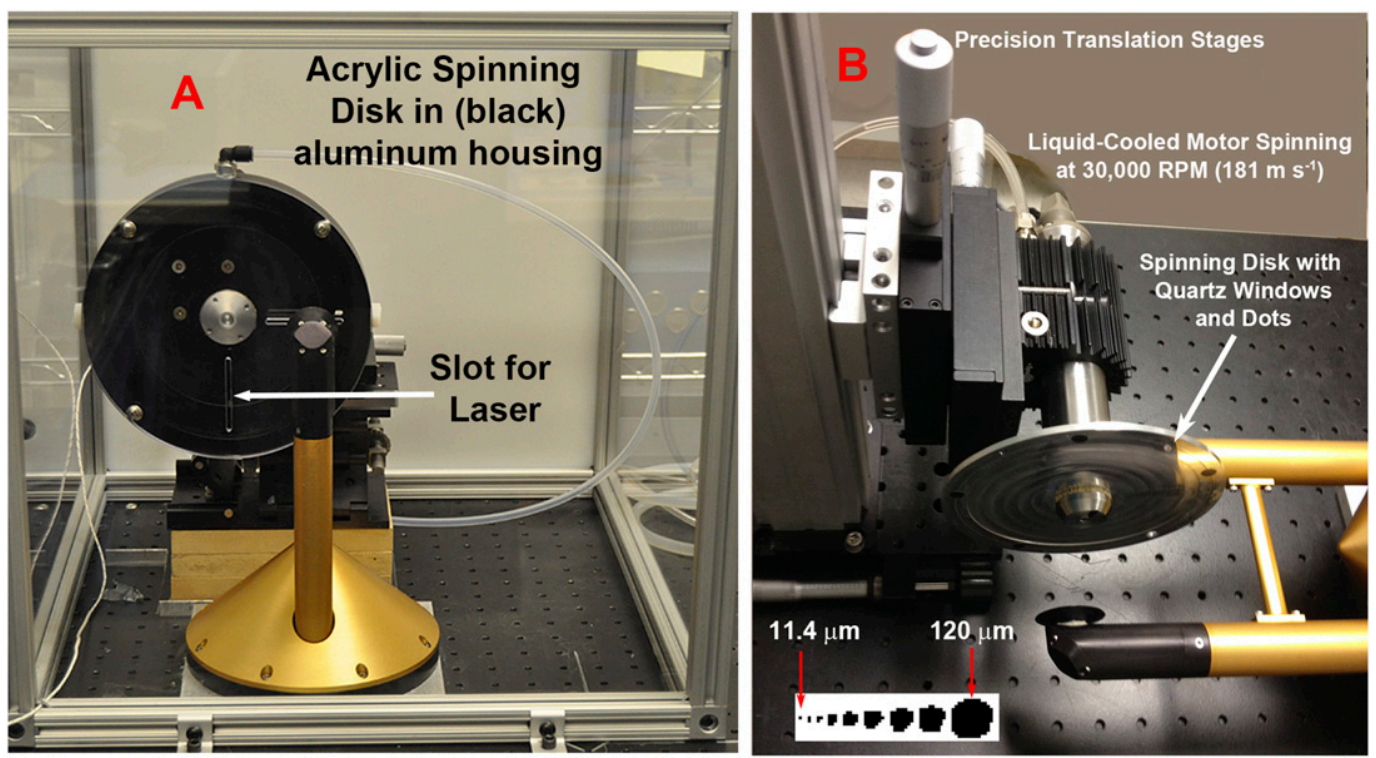

FIG. 5. Photographs of (a) an acrylic disk in protective aluminum housing and (b) aluminum disk with small clear sapphire windows. Inset shows 2D-128 images of dots from 11.4 to $120 \mu \mathrm{m}$. 


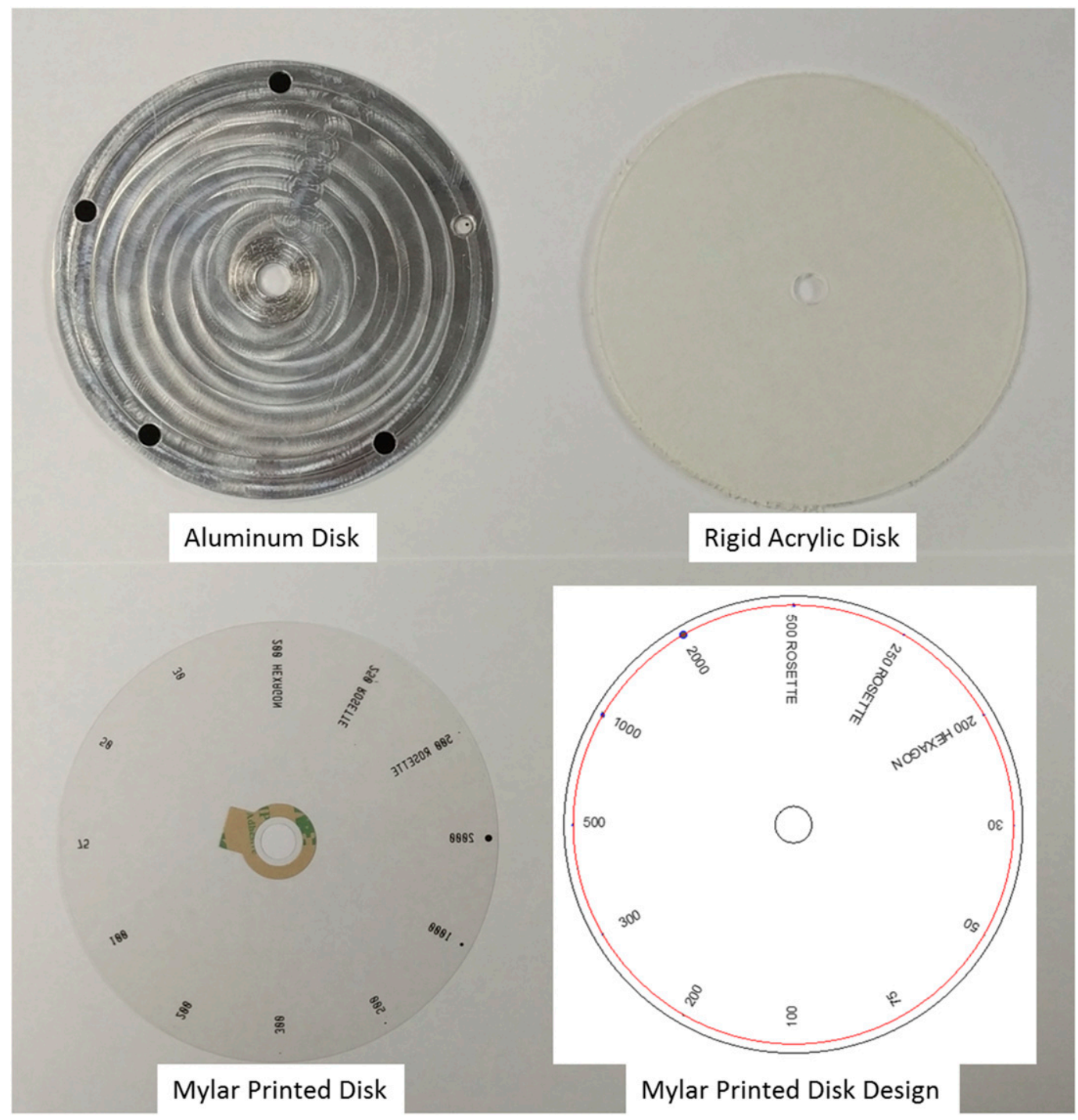

FIG. 6. Images of the high-speed spinning disks utilized in this study. The disks have a 10-mm spindle hole in the middle, an outer diameter of $125 \mathrm{~mm}$ (aluminum and Mylar disks), and $120 \mathrm{~mm}$ for the acrylic disk. The aluminum disk contains a $0.5-\mathrm{cm}$ reticle window with printed opaque dot features. Similarly, the Mylar disk has uniformly spaced opaque features (dots of increasing diameter, rosettes, and a hexagon) printed at a radius of $120 \mathrm{~mm}$ (all printed features lie on the red line in the printed disk design schematic). The aluminum and acrylic disks are considered rigid because they do not "wobble" at high speeds. The thinner Mylar disk is observed to wobble at higher speeds, but the advantage of the Mylar disks is that they can maintain speeds of $\sim 200 \mathrm{~m} \mathrm{~s}^{-1}$ for a prolonged period, which is challenging for the heavier aluminum and acrylic disks, because of their increased weight.

number of pixels corresponding to each of the dots $\leq$ $1000 \mu \mathrm{m}$ varies by about one pixel for both the $2 \mathrm{D}-128$ and CIP02, which can be expected as a result of a combination of disk position and digitization effects. The effect of digitization on the formation of binary digital images has been discussed in Korolev et al. (1998). Uncertainties in the sizes of the dot diameters $(D)$ as measured using a high-magnification microscope and reticle are $30 \leq D \leq 75 \mu \mathrm{m}=1.5 \mu \mathrm{m}$ and
$100 \leq D \leq 1 \mathrm{~mm}=2.5 \mu \mathrm{m}$. The data also suggest that the actual pixel size of the $2 \mathrm{D}-128$ is about $11.1 \mu \mathrm{m}$, and the actual pixel size of the CIP02 is about $27 \mu \mathrm{m}$. The difference in the nominal compared with actual pixel size is generally attributed to tolerance in the lens specifications.

The Mylar disk with printed images was moved across the depth of field using the micropositioner to determine the smallest dot that the CIP can image as a 
TABLE 1. Number of 2D-128 and CIP02 pixels for various dots imaged on the Mylar disk and recorded as a function of disk speed.

\begin{tabular}{ccccccccc}
\hline \hline \multicolumn{7}{c}{ Dot size $(\mu \mathrm{m})$} \\
\hline Disk & 1000 & 500 & 300 & 200 & 100 & 75 & 50 & 30 \\
\cline { 2 - 8 } speed (rpm) & \multicolumn{7}{c}{ 2D-128 No. of $10-\mu$ pixels $^{\mathrm{a}}$} \\
\hline 6000 & 89 & 45 & 28 & 19 & 10 & 7 & 5 & 3 \\
12000 & 89 & 46 & 28 & 20 & 10 & 7 & 5 & 3 \\
18000 & 87 & 44 & 26 & 18 & 9 & 7 & 5 & 3 \\
24000 & 89 & 46 & 28 & 20 & 9 & 8 & 5 & 3 \\
30000 & 89 & 46 & 28 & 20 & 10 & 7 & 4 & 3 \\
7 & CIP02 No. of $25-\mu$ m pixels ${ }^{\mathrm{a}}$ & & & \\
\hline 6000 & 37 & 19 & 11 & 7 & 4 & 3 & 2 & 1 \\
12000 & 37 & 18 & 11 & 8 & 4 & 3 & 2 & 1 \\
18000 & 38 & 19 & 12 & 8 & 3 & 3 & 2 & 1 \\
24000 & 38 & 19 & 12 & 7 & 4 & 3 & 2 & 1 \\
30000 & 37 & 19 & 11 & 7 & 4 & 3 & 2 & 1 \\
\hline
\end{tabular}

${ }^{\mathrm{a} N o m i n a l ~ p i x e l ~ s i z e ~ t h a t ~ m a y ~ v a r y ~ s l i g h t l y ~ f r o m ~ p r o b e ~ t o ~ p r o b e . ~}$

function of disk speed. Table 1 shows that CIP02 images the $30-\mu \mathrm{m}$ dot and the pixel size of each of the larger dots to within about one pixel at all disk speeds. However, it was extremely difficult to detect the $30-\mu \mathrm{m}$ dot with both CIP02 and CIP17. Very precise positioning with micro translation stages was required. It appeared that the dot could only be detected when it precisely occulted a very small percentage of photodiodes on the array. This suggests that perhaps the responsivity of photodiodes or the detection threshold levels differ from photodiode to photodiode. A similar result has been suggested by Hayman et al. (2016) in their analysis of the time response of CIP electronics.

Figure 7 shows images on the Mylar disk recorded at $38,76,114,152$, and $190 \mathrm{~m} \mathrm{~s}^{-1}$ by the $2 \mathrm{D}-128$ using the SPEC 2D-S data system. The disk was moved along the laser beam perpendicular to the arms of the probe ( $z$ axis) using a micropositioner to determine the smallest resolvable feature at each disk speed. The effective concentration of the printed disk at $10-\mu \mathrm{m}$ resolution is about $100 \mathrm{~cm}^{-3}$, which is similar to the upper end of the drop concentration in maritime cumulus clouds (Pruppacher and Klett 1997) and Arctic stratus clouds (Hobbs and Rangno 1998; Lawson and Zuidema 2009). This volumetric estimate is derived by assuming that the particles on the disk are distributed uniformly between the arms of the probe. The 2D-128 sees all of the images printed on the disk at a range of tangential disk velocities from

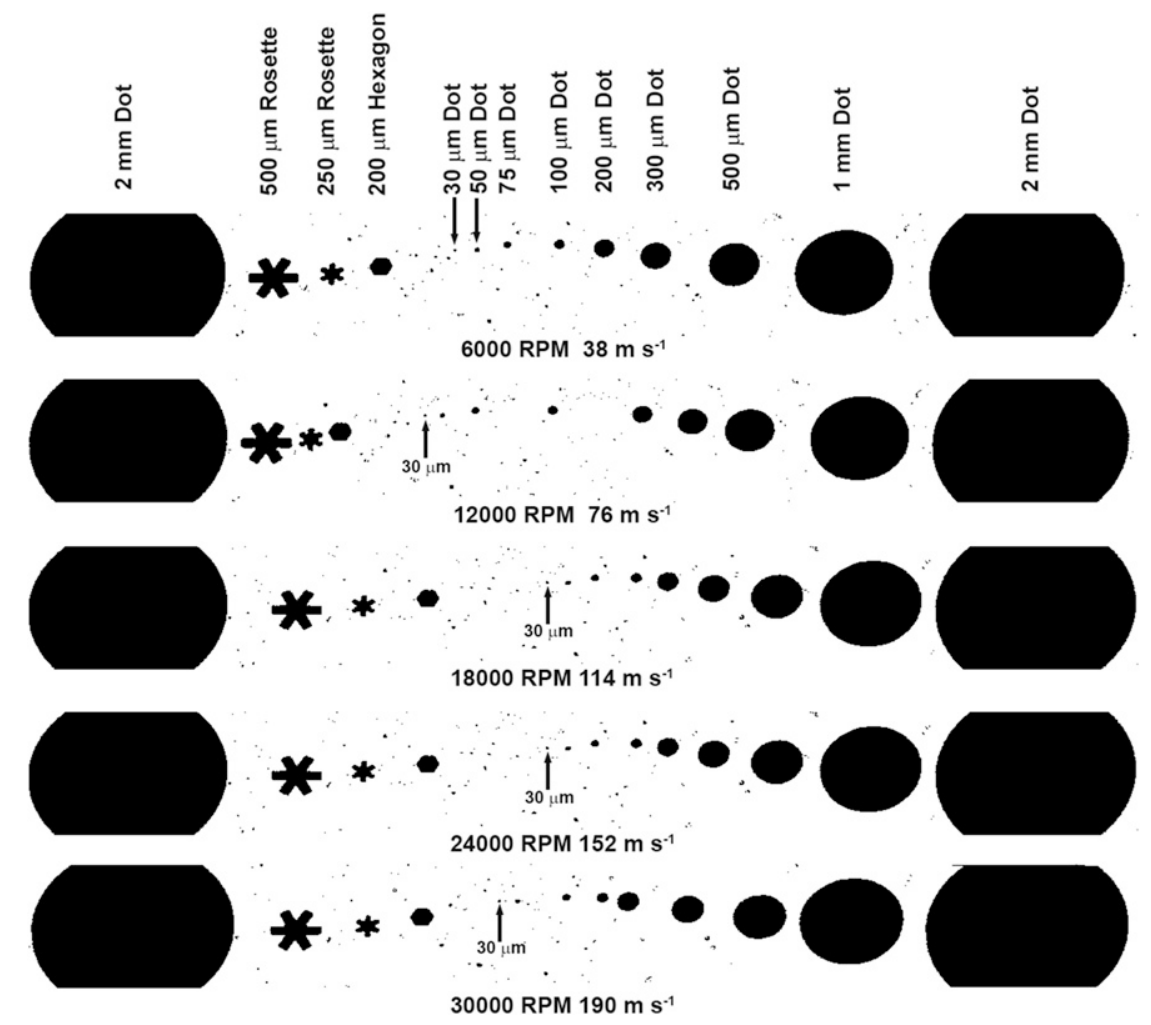

FIG. 7. Images of features on the Mylar disk recorded during one revolution by the 2D-128 at various disk speeds. The $2-\mathrm{mm}$ dot is repeated for reference. 


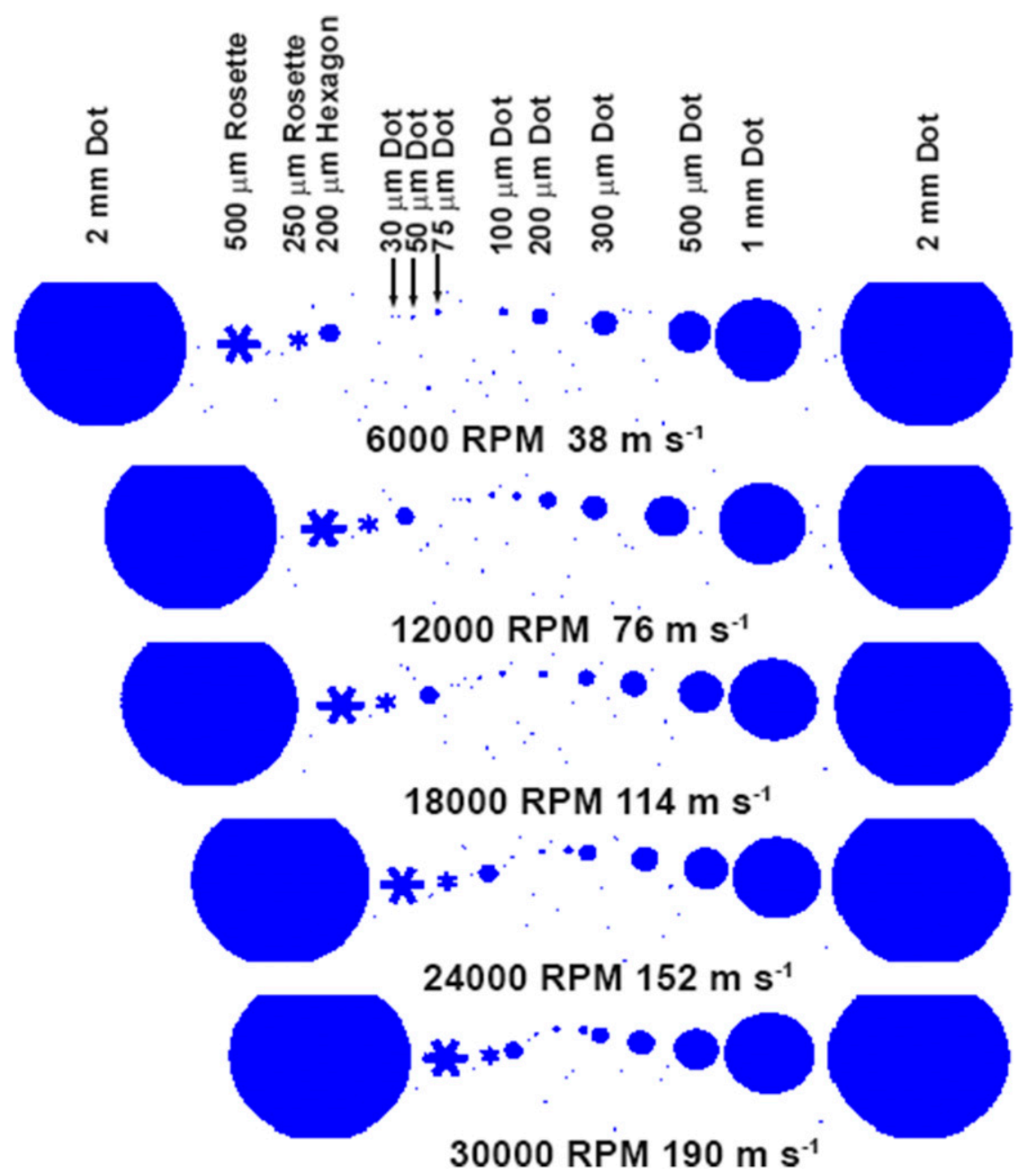

FIG. 8. As in Fig. 7, but for images recorded by the CIP02.

38 to $190 \mathrm{~m} \mathrm{~s}^{-1}$. $^{2}$ Visually, Fig. 7 shows there is no degradation in the number of particles imaged by the 2D-128 at disk speeds ranging from 38 to $190 \mathrm{~m} \mathrm{~s}^{-1}$. We use the qualitative results shown in Fig. 7 as a benchmark to compare the CIPs. We show more quantitative results later in this section using the rigid disk and flight data.

CIP02 and CIP17 probes were tested with the same Mylar disk used to test the 2D-128. Figures 8 and 9 show images from these two probes in the same format as Fig. 7, recorded using the DMT Particle Analysis and Display System (PADS) data system and analyzed using NCAR System for Optical Array Probe Data Analysis

\footnotetext{
${ }^{2}$ We refer subsequently to the tangential disk rotation speed as disk speed, which is analogous to true airspeed on an aircraft. When imaging the 30-, 50-, and 75- $\mu \mathrm{m}$ dots, the position of the disk is moved in $0.1-\mu \mathrm{m}$ increments across the DOF to find the position of best focus for each individual dot.
}

(SODA) software. ${ }^{3}$ The nominal resolution of the CIP is $25 \mu \mathrm{m}$, so surface defects smaller than about $25 \mu \mathrm{m}$ cannot be imaged by these probes. The striking feature seen in Figs. 8 and 9 is how the total number of smaller $(<75 \mu \mathrm{m})$ images decreases with disk speed. This is in general agreement with the theoretical work presented in Baumgardner and Korolev (1997) and Korolev et al. (1998). This set of papers modeled the effects of particle diffraction on the photodiode array coupled with response time of the front-end electronics. This combination of factors effectively reduces shadow depth and causes missing and undersized one- to four-pixel particles. As pointed out in Korolev et al. (1998, p. 712), "smaller

\footnotetext{
${ }^{3} \mathrm{CIP}$ particle concentrations and size distributions shown in this paper were computed using the SODA code developed by Aaron Bansemer at the National Center for Atmospheric Research (NCAR).
} 


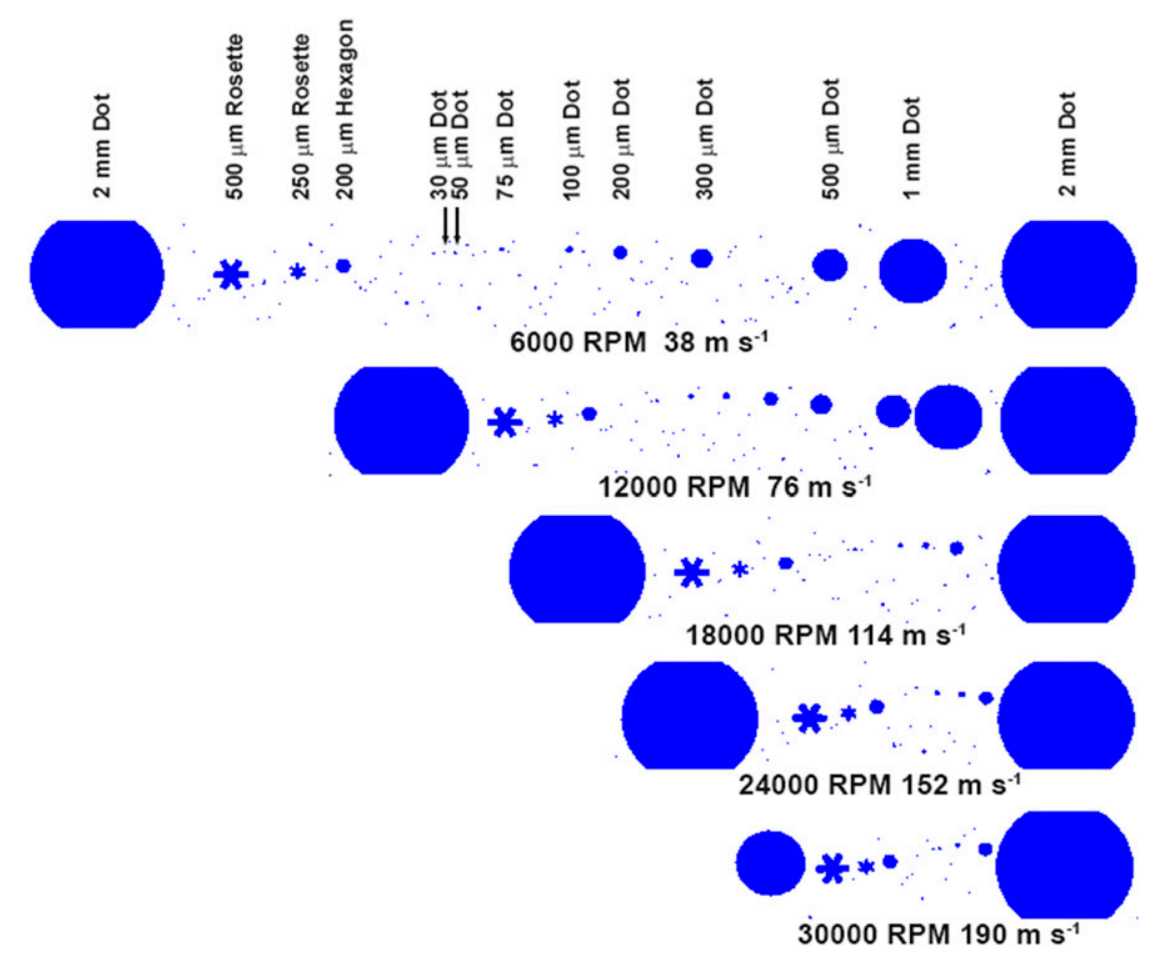

FIG. 9. As in Fig. 7, but for images recorded by the CIP17.

photodiodes spaced at regular intervals provide superior discrete images for particle sizing and shape definition than larger photodiodes with the same spacing." The CIPs have photodiodes that are individually masked with a $300-\mu \mathrm{m}$ diameter circular window; the $2 \mathrm{D}-\mathrm{S}$ probe has pixels that are $42.5 \mu \mathrm{m} \times 50 \mu \mathrm{m}$. The loss of small particles in Figs. 8 and 9 with increasing disk speed as a result of inadequate time response is not observed in Fig. 7.

While Figs. 8 and 9 show the effects of slower time response of the CIPs compared with the 2D-128 (i.e., 2D-S), Figs. 10 and 11 show the effects of dead time on CIP02 and CIP17, respectively. In each figure, six consecutive 4096-byte buffers are shown when the Mylar disk was spun at $38 \mathrm{~m} \mathrm{~s}^{-1}(6000 \mathrm{rpm})$ and at $190 \mathrm{~m} \mathrm{~s}^{-1}$ (30000 rpm). Figure 10 shows that the number of images recorded by the 16-bit particle counter ("Part. Ctr." shown on the left side of each buffer) exactly equals the number of 2D images in the buffer ("2D Imgs." shown below Part. Ctr.) at $38 \mathrm{~m} \mathrm{~s}^{-1}$. However, at $190 \mathrm{~m} \mathrm{~s}^{-1}$, the number of actual 2D images in alternating buffers is about $50 \%$ of the number of images recorded by the particle counter. The missed images (Part. Crt. minus 2D Imgs.) are due to dead time within the buffer. The location where the probe went into overload can be seen by the fragmented images in the red boxes and the discontinuous series of images that follow (i.e., images that do not follow the pattern of images printed on the disk, such as seen in Fig. 7). Figure 11 is shown in the same format as Fig. 10 but for the CIP17 probe. Unfortunately, the more modern CIP17 probe suffers significantly more dead time than CIP02, experiencing missing images within all buffers, even at $38 \mathrm{~m} \mathrm{~s}^{-1}$.

Since the CIPs size the printed images to within one pixel and image the $30-\mu \mathrm{m}$ dot at $190 \mathrm{~m} \mathrm{~s}^{-1}$, the question begs to be asked, Why do the CIPs appear to miss so many of the small (one and two pixels) permanent surface defects in Figs. 8 and 9? To investigate the decrease in small particles seen by both CIPs as the disk speed increases and to eliminate the complicating influence of dead time, the thicker, "rigid" acrylic disk without large printed images and with a relatively low concentration of small defects was used. Dead time was not an issue with the CIP02 probe. Wobble from the thicker acrylic disk is nonexistent, as verified by photographing the rotating disk. The maximum disk speed of the thicker disk was $176 \mathrm{~m} \mathrm{~s}^{-1}$ as a result of the increased motor loading. The concentration of permanent surface defects was determined by exposing the disk under a microscope lens system and recording the data using a digital camera. The images were then analyzed using software developed by the National Institutes of Health called ImageJ (https://imagej.nih.gov/ij/), which provides the area in number of pixels for each particle. An 

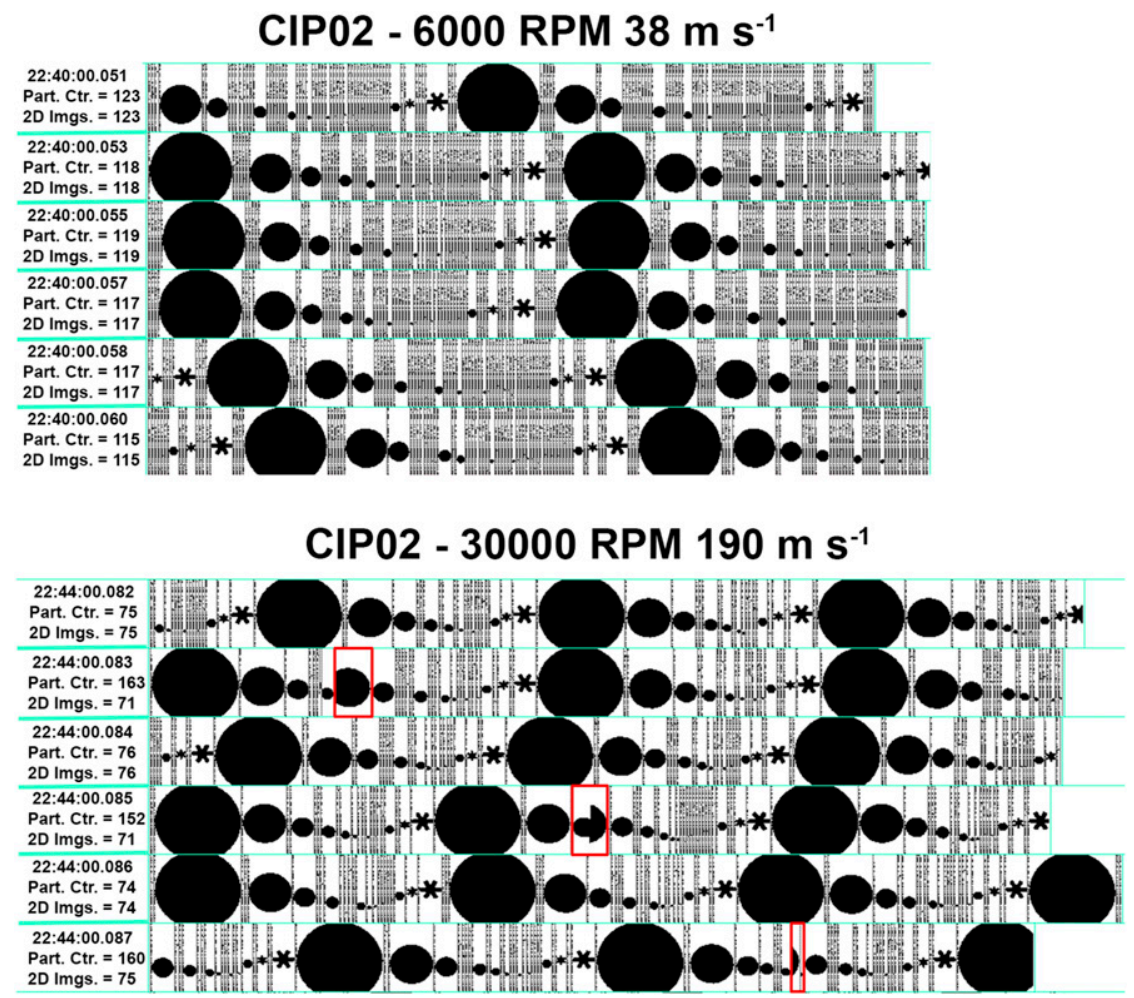

FIG. 10. Each image strip is one CIP02 buffer recorded at (top) 38 and (bottom) $190 \mathrm{~m} \mathrm{~s}^{-1}$. Red boxes show places in the buffer where the probe went into overload, where particles were missed, and where dead time occurred but was not recorded.

example of typical images of the surface defects taken with a digital camera are shown in Fig. 12a. The same images digitized at a $50 \%$ threshold into black-andwhite images are shown in Fig. 12b. The average particle size distribution and mean concentrations for particles $>10 \mu \mathrm{m}$ and particles $>25 \mu \mathrm{m}$ are shown in Fig. 12c.

Figures 13-15 show images recorded in one disk revolution as a function of disk speed from the 2D-128, CIP02, and CIP17, respectively. An identifying mark on the disk was used to determine one disk revolution. Table 2 shows the number of 2D particles per revolution from the 2D-128, CIP02, and CIP17 recorded as a function of disk speed. The 1D particle count is also shown. As shown in Fig. 13 and Table 2, the 2D-128 data system records a nearly constant number of images at all disk speeds. For example, 245 images are recorded at $44 \mathrm{~m} \mathrm{~s}^{-1}$ and 246 images are recorded at $176 \mathrm{~m} \mathrm{~s}^{-1}$. The variation in the number of images at intermediate disk speeds is due to slight movement of the disk across the field of view, which is caused by slight radial deformation of the disk at higher speeds. The acrylic disk moves about $40 \mu \mathrm{m}$ perpendicular to the direction of travel. This causes some defects to move out of the field of view, while others may move into the field of view. This makes a one-to-one comparison of the small defects intractable as disk speed increases. However, averaging the number of defects over several revolutions gives a good statistical average of the number of defects imaged.

Table 2 shows that the number of $2 \mathrm{D}$ images recorded by the CIP02 data system decreases monotonically as a function of disk speed: $65 \%$ of the number of images recorded at $44 \mathrm{~m} \mathrm{~s}^{-1}$ are recorded at $176 \mathrm{~m} \mathrm{~s}^{-1}$. The CIP17 probe shows a similar trend in the number of images recorded by the data system as a function of disk speed, with $50 \%$ of the images recorded at $176 \mathrm{~m} \mathrm{~s}^{-1}$ compared to the number recorded at $44 \mathrm{~m} \mathrm{~s}^{-1}$ (Table 2). As seen in Table 2, the $1 \mathrm{D}$ particle counts also decrease with increasing disk speed at about the same rate as the $2 \mathrm{D}$ counts. The data rate of this relatively low concentration of small particles is not high enough to drive CIP02 into overload, so the missing particles are presumably due to the inadequate time response of the electronics coupled with digitization effects on the photodiode array (Baumgardner and Korolev 1997; Korolev et al. 1998). Korolev et al. (1998) found theoretically that up to $60 \%$ of one-pixel particles can be 

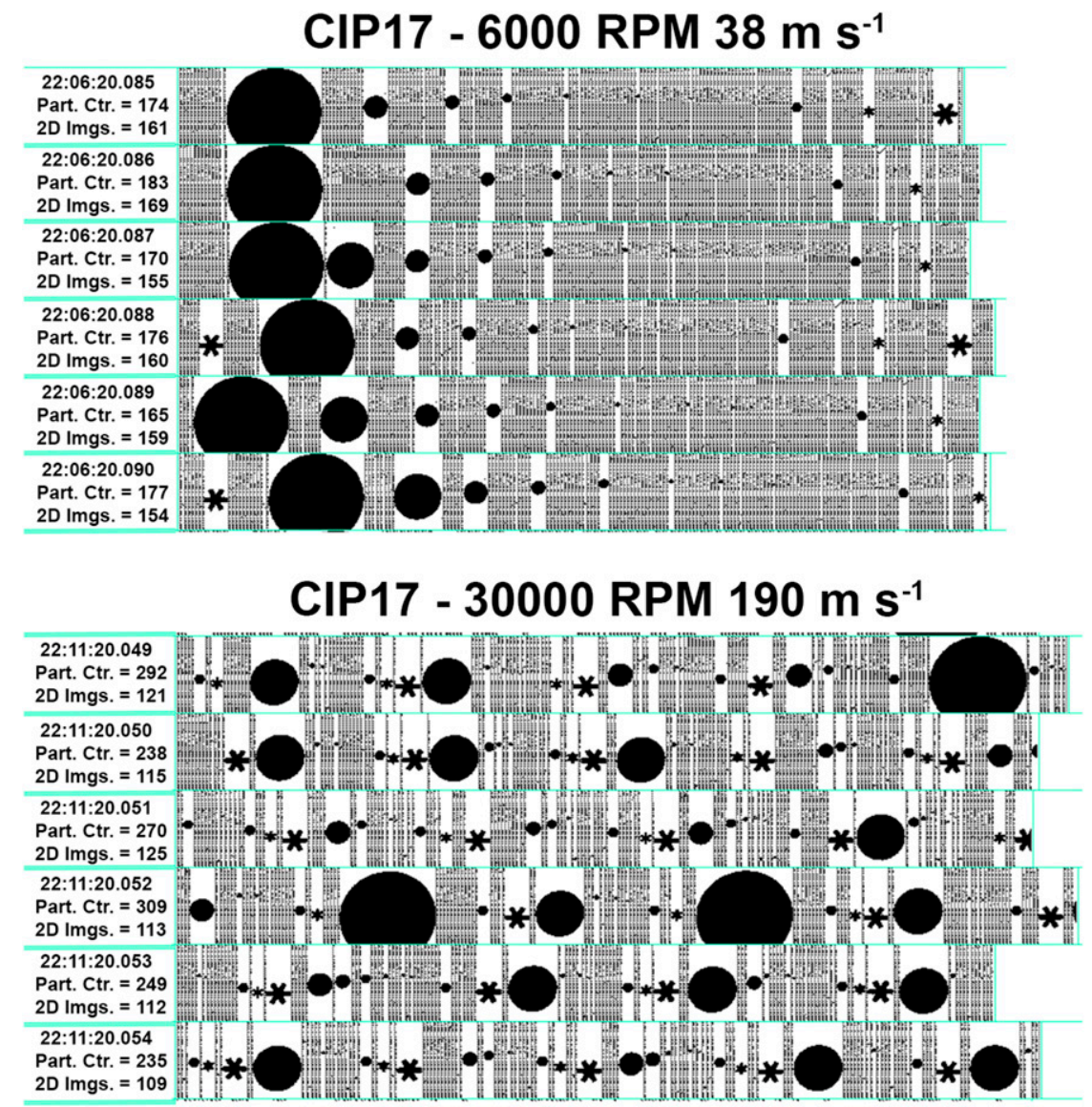

FIG. 11. Each image strip is one CIP17 buffer.

missed as a result of digitization effects of the photodiode array used in the 2D-C probe. Hayman et al. (2016) tested the front-end electronics of a CIP and found significant variation in the response characteristics of the different photodiode circuits. They also found a slow decay term that can impact the probe response, concluding that the greatest sensitivity to response time characteristics is for particles smaller than $150 \mu \mathrm{m}$.

\section{Flight test with both CIP and 2D-S}

CIP02 and a 2D-S probe were installed on the SPEC Learjet to see whether the results from the spinning disk tests could be substantiated with flight tests. The 2D-S and $2 \mathrm{D}-128$ probes process particle data using only $2 \mathrm{D}$ images. The CIP records $2 \mathrm{D}$ images, but these are not analyzed quantitatively in some applications. The SODA code can be used to analyze CIP 2D images, but according to the manufacturer, the $1 \mathrm{D}$ output is used for generating real-time and quick-look particle concentration and size distributions (M. Freer 2017, personal communication).
The effect of disk speed on the response of the 2D-128 probe and CIP was shown in Figs. 7-11 and 13-15 and Tables 1 and 2. Both the CIP02 probe and a SPEC 2D-S were installed on the SPEC Learjet and flown in a warm stratus cloud on 5 September 2017 that also contained large ash particles from pervasive wild fires in Colorado, Montana, and California. The result was a cloud with particles ranging in size from about 5 to $80 \mu \mathrm{m}$, similar to the sizes of the surface defects on the rigid disk (see Figs. 12, 13). Based on 2D-S measurements, two 10-s regions of cloud with about the same average particle concentration $\left(\sim 20 \mathrm{~cm}^{-3}\right)$ were penetrated at 99 and $170 \mathrm{~m} \mathrm{~s}^{-1}$.

Figure 16a shows a time series of particle concentration from the 2D-S and CIP02 2D SODA code at 99 and $170 \mathrm{~m} \mathrm{~s}^{-1}$. Figure $16 \mathrm{~b}$ shows size distributions from the $2 \mathrm{D}-\mathrm{S}$ in $10-$ and $25-\mu \mathrm{m}$ size bins (to compare with the CIP02) at $99 \mathrm{~m} \mathrm{~s}^{-1}$. Figure $16 \mathrm{c}$ shows size distributions at $170 \mathrm{~m} \mathrm{~s}^{-1}$. The 2D-S records a much higher average concentration than the CIP02, which is expected as a result of its higher $(10 \mu \mathrm{m})$ pixel resolution compared to the $(25 \mu \mathrm{m})$ CIP pixel resolution. However, as seen in 


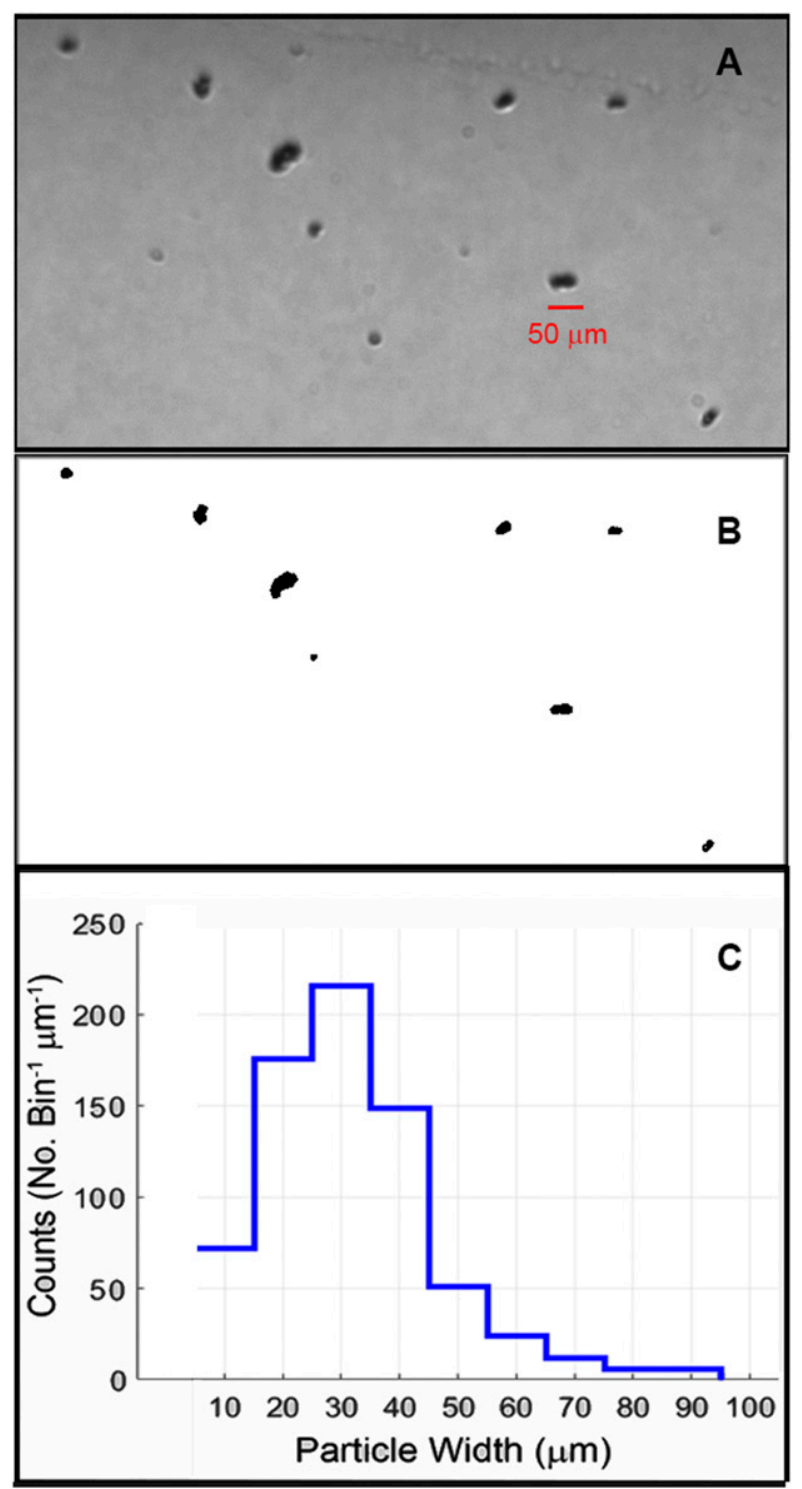

FIG. 12. (a) Images on acrylic disk recorded with a digital camera. (b) Digitized at $50 \%$ threshold. (c) Size distribution.

the time series (Fig. 16a), the average concentration of the $2 \mathrm{D}-\mathrm{S}$ is $19390 \mathrm{~L}^{-1}$ at $99 \mathrm{~m} \mathrm{~s}^{-1}$ and 16587 at $170 \mathrm{~m} \mathrm{~s}^{-1}$ ( $86 \%$ of the value at $99 \mathrm{~m} \mathrm{~s}^{-1}$ ), whereas the average CIP concentration at $170 \mathrm{~m} \mathrm{~s}^{-1}\left(19 \mathrm{~L}^{-1}\right)$ is $9 \%$ of the value at $99 \mathrm{~m} \mathrm{~s}^{-1}\left(213 \mathrm{~L}^{-1}\right)$. This is partially attributed to a higher concentration of $25-\mu \mathrm{m}$ particles in the cloud pass at $170 \mathrm{~m} \mathrm{~s}^{-1}$. The data in Fig. 16 are more readily quantified by the size distributions shown on linear scales in Figs. $17 \mathrm{c}$ and $17 \mathrm{~d}$, where the $2 \mathrm{D}-128$ data have again been interpolated into $25-\mu \mathrm{m}$ size bins for easier comparison with the CIP02. However, while the number of $25-\mu \mathrm{m}$ particles has decreased from 3575 at $99 \mathrm{~m} \mathrm{~s}^{-1}$ to $2250(63 \%)$ at $170 \mathrm{~m} \mathrm{~s}^{-1}$, the number of CIP02 particles

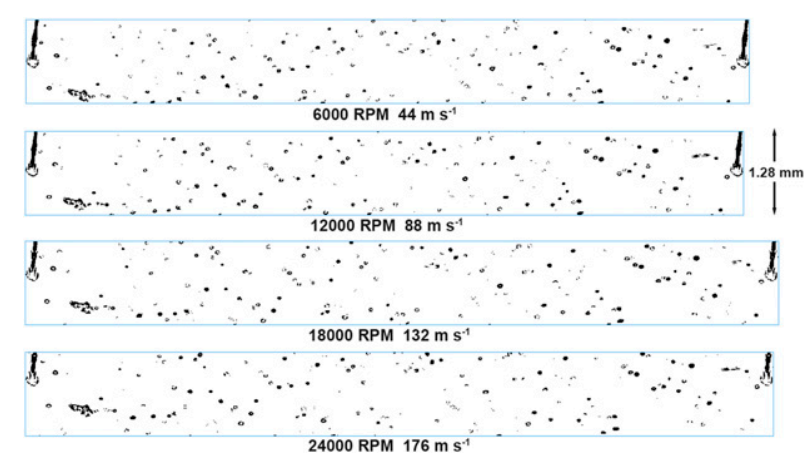

FIG. 13. 2D-128 images recorded in one revolution on the rigid acrylic disk as a function of disk speed. Larger vertical scratch is repeated for reference.

has decreased from 1800 at $99 \mathrm{~m} \mathrm{~s}^{-1}$ to $250(14 \%)$ at $170 \mathrm{~m} \mathrm{~s}^{-1}$. Note that here we use the CIP 1D $25-\mu \mathrm{m}$ particle count, which is always higher than the CIP 2D particle count and because the SODA code automatically combines the $25-$ and $50-\mu \mathrm{m}$ bins.

Since there was no dead time experienced by the CIP02 probe during the flight data, it is apparent that the decrease in detection efficiency of the CIP02 probe is due to inadequate time response coupled with digitization of diffraction effects on the photodiode array. The percentage of small particles missed by CIP02 is more severe than the results using the spinning disk shown in Table 2, where the CIP02 and CIP17 2D counts at $176 \mathrm{~m} \mathrm{~s}^{-1}$ were $65 \%$ and $50 \%$, respectively, of the counts at $88 \mathrm{~m} \mathrm{~s}^{-1}$. The lower imaging efficiency of the CIP during the Learjet flight is probably due to the higher percentage of one-pixel $(25 \mu \mathrm{m})$ particles in the cloud compared with the disk, which contained relatively more two- and three-pixel particles.

\section{Summary and discussion}

The primary objective of this research is to educate investigators regarding the efficacy of data collected by 2D imaging probes commonly used on research aircraft. Unlike papers that have focused only on the time response of the front-end electronics of these probes (e.g., Baumgardner and Korolev 1997; Strapp et al. 2001; Hayman et al. 2016), our work elucidates the response characteristics of the entire chain of electro-optics in the probes tested. Examination of the complete throughput of the $2 \mathrm{D}$ imaging probes has revealed measurement uncertainties that investigators can take into account when incorporating CIP $1 \mathrm{D}$ data and 2D imaging data into their results.

We examined the response characteristics of two CIPs, one manufactured in 2002 (CIP02) and a second 

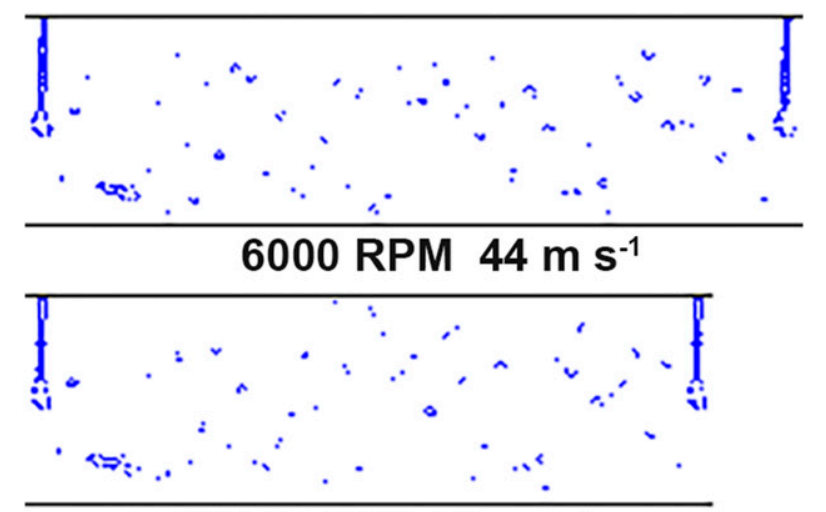

12000 RPM $88 \mathrm{~m} \mathrm{~s}^{-1}$

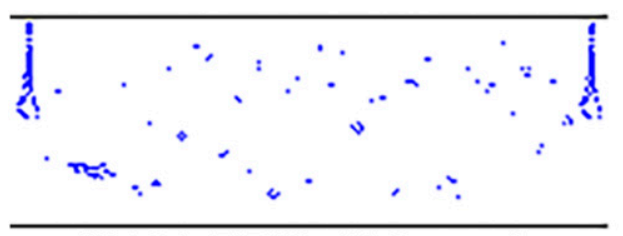

18000 RPM $132 \mathrm{~m} \mathrm{~s}^{-1}$

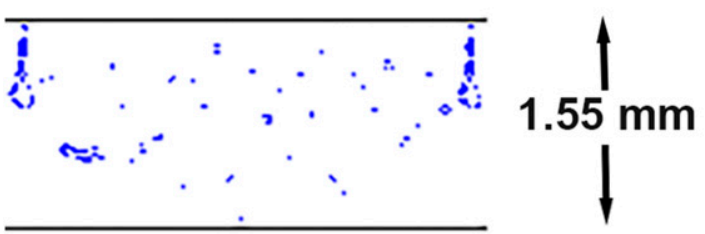

24000 RPM $176 \mathrm{~m} \mathrm{~s}^{-1}$

FIG. 14. As in Fig. 13, but for CIP02 images.

manufactured in 2017 (CIP17), and a 2D-128 probe manufactured in 2007 . The $2 \mathrm{D}-128$ is a single-channel 2D-S probe. The DOF of each probe was examined using a drop generator that projected drops with diameters accurate to $1 \mu \mathrm{m}$ through the sample areas of the probes (see the appendix for details). The primary tools used to evaluate the performance of each probe are a transparent Mylar spinning disk with (30-2000 $\mu \mathrm{m})$ opaque dots, a hexagon and two different sized rosettes (Fig. 6), and a more rigid acrylic disk. The Mylar disk is capable of translational speeds up to $190 \mathrm{~m} \mathrm{~s}^{-1}$. The acrylic disk is thicker and more rigid, and it contained only small permanent surface defects with irregular shapes. The defects are from about 10 to $100 \mu \mathrm{m}$ in size, with the large majority being $\leq 50 \mu \mathrm{m}$ (Fig. 12). One custom aluminum disk with small clear sapphire windows with dots from 11.5 to $120 \mu \mathrm{m}$ was used to confirm that the 2D-128 could image the $11.4-\mu \mathrm{m}$ dot at $181 \mathrm{~m} \mathrm{~s}^{-1}$. This result supports measurements reported by Lawson et al. (2006) showing that the 2D-S imaged an $8-\mu \mathrm{m}$-diameter glass fiber spinning at $233 \mathrm{~m} \mathrm{~s}^{-1}$. The aluminum disk would not work for the CIPs. This is because the much longer-duration occultation produced

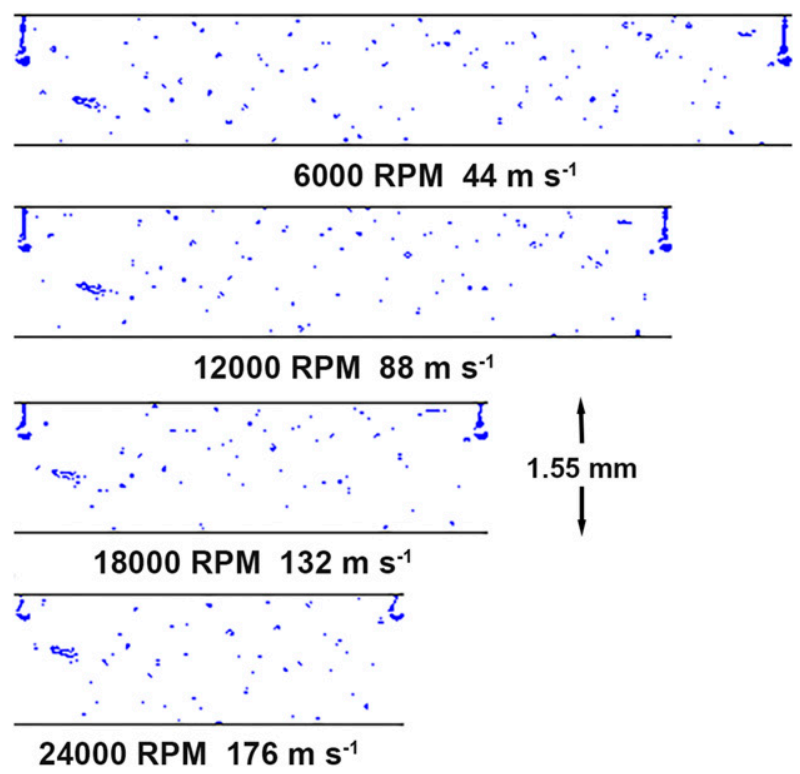

FIG. 15. As in Fig. 13, but for CIP17 images.

by the opaque aluminum did not allow the electronics to reset in time to see the small sapphire windows.

The performance of the 2D-128 and the CIPs was evaluated over a speed range from 38 to $190 \mathrm{~m} \mathrm{~s}^{-1}$ using the (thin) Mylar disk with the printed images, and from 44 to $176 \mathrm{~m} \mathrm{~s}^{-1}$ using the thicker rigid acrylic disk. The rigid disk is heavier, so it could not reach the disk speed of the thin acrylic disk. Both the 2D-128 and CIPs sized in-focus 30-1000- $\mu \mathrm{m}$ printed dots onto the Mylar disks to within about one pixel throughout the disk speed range from 38 to $190 \mathrm{~m} \mathrm{~s}^{-1}$ (Table 1). However, it was extremely tedious to locate the $30-\mu \mathrm{m}$ dot using the CIPs. This may be due to variance in the sensitivity and thresholding of photodiodes in the 64-element array, as suggested by Hayman et al. (2016).

Both the Mylar and rigid acrylic disks move slightly $(\sim 40 \mu \mathrm{m})$ perpendicular to the direction of rotation as disk speed increases, so the disk sample area changed slightly at the periphery of the array. Also, the Mylar disk with the printed images wobbles as the speed increases, so a one-pixel defect may go out of focus and disappear while another may come into focus and appear. A larger defect may appear smaller when out of focus. The wobble of the Mylar disk and movement made it impossible to map a one-to-one comparison of small images onto the disk as the disk speed changes. However, since the effect of wobble is random and the defects on the disk are distributed randomly, averaging over several revolutions provides robust statistics. The rigid acrylic disk was photographed and found not to wobble. 
TABLE 2. Number of 2D (and 1D CIP) images recorded as a function of disk speed for 2D-128 and CIPs.

\begin{tabular}{|c|c|c|c|c|c|c|}
\hline \multirow[b]{2}{*}{ Disk speed (rpm) } & \multirow[b]{2}{*}{ Tangential velocity $\left(\mathrm{m} \mathrm{s}^{-1}\right)$} & \multicolumn{3}{|c|}{$2 \mathrm{D}$ images one full rotation particle count } & \multicolumn{2}{|c|}{$\begin{array}{l}1 \mathrm{D} \text { images one full } \\
\text { rotation particle count }\end{array}$} \\
\hline & & $2 \mathrm{D}-128$ & CIP02 & CIP17 & $\overline{\mathrm{CIP} 02}$ & CIP17 \\
\hline 6000 & 44 & 245 & 72 & 114 & 56 & 93 \\
\hline 12000 & 88 & 239 & 66 & 98 & 50 & 78 \\
\hline 18000 & 132 & 269 & 57 & 72 & 40 & 63 \\
\hline 24000 & 176 & 246 & 47 & 57 & 29 & 51 \\
\hline
\end{tabular}

Tests with the Mylar disk spun at speeds from 38 to $190 \mathrm{~m} \mathrm{~s}^{-1}$ were conducted to evaluate the effects of dead time on the measurements. The 2D-128 did not experience significant sequential particle loss at any disk speed (Fig. 7). Visual evidence of image loss showed that both CIP02 and CIP17 go into overload and suffer dead time within the fixed-length 4096-byte buffers at $190 \mathrm{~m} \mathrm{~s}^{-1}$ (Figs. 10, 11). However, the more modern CIP17 was much more susceptible, experiencing dead time at speeds as low as $38 \mathrm{~m} \mathrm{~s}^{-1}$. CIP02 does not go into overload and experiences dead time until a threshold data rate is reached, which is about 100 buffers per second. For reference, this corresponds to about $350 \mathrm{~L}^{-1}$ of $100-\mu \mathrm{m}$ drops, having a liquid water content of about $0.2 \mathrm{~g} \mathrm{~m}^{-3}$. In a comparison, using the Mylar disk with printed images, at $190 \mathrm{~m} \mathrm{~s}^{-1}$ CIP02 missed about $50 \%$ of the particles in every other buffer as a result of dead time losses, and the CIP17 missed about $45 \%$ of the particles in every buffer, with a large variance between buffers.
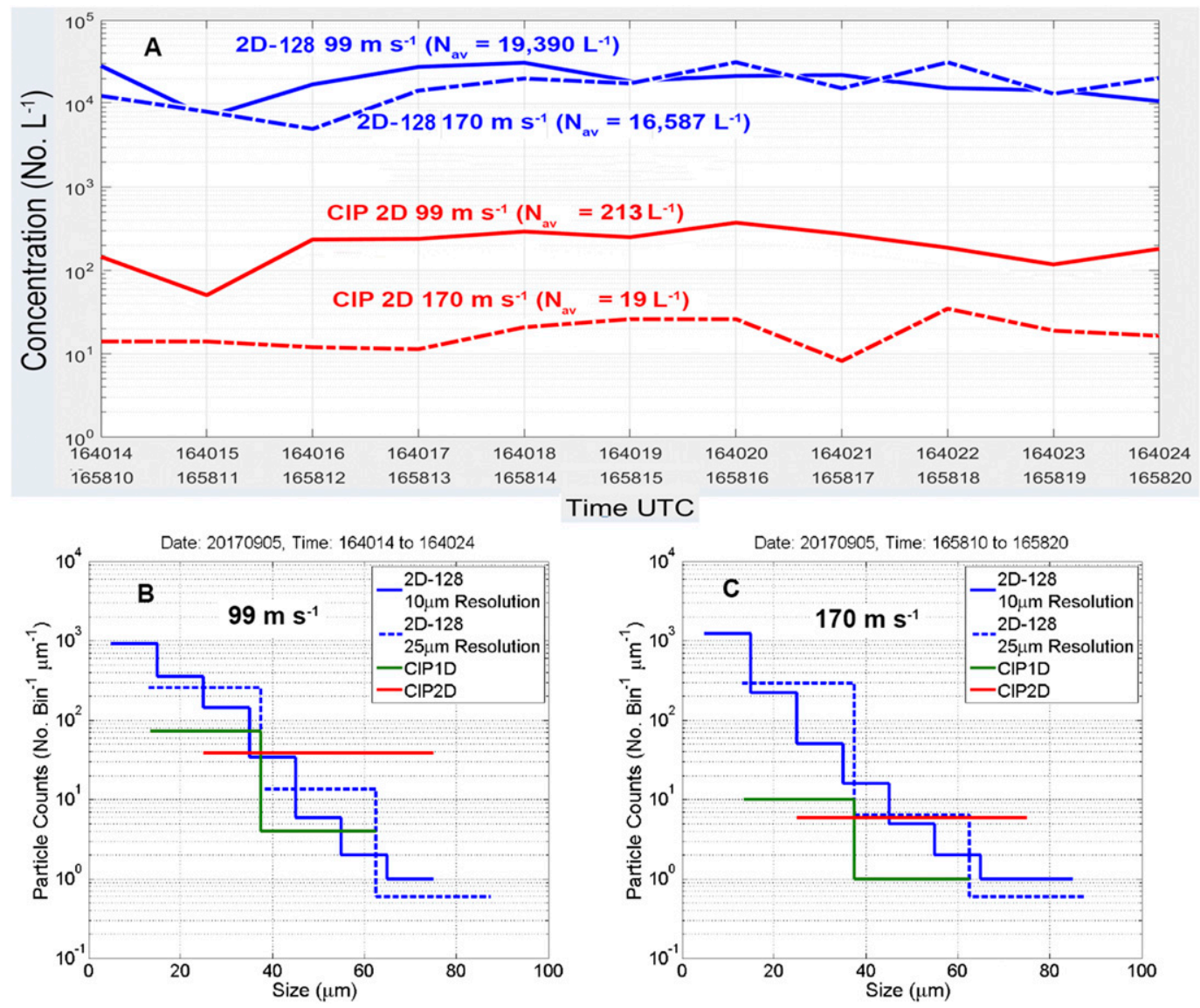

FIG. 16. (a) 2D-128 and CIP02 time series of total particle concentration measured at Learjet true airspeed of 99 and $170 \mathrm{~m} \mathrm{~s}^{-1}$. Particle size distributions at (b) 99 and (c) $170 \mathrm{~m} \mathrm{~s}^{-1} .2 \mathrm{D}-128$ interpolated to $25-\mu \mathrm{m}$ size bins to compare with the CIP02 (dashed blue line). 

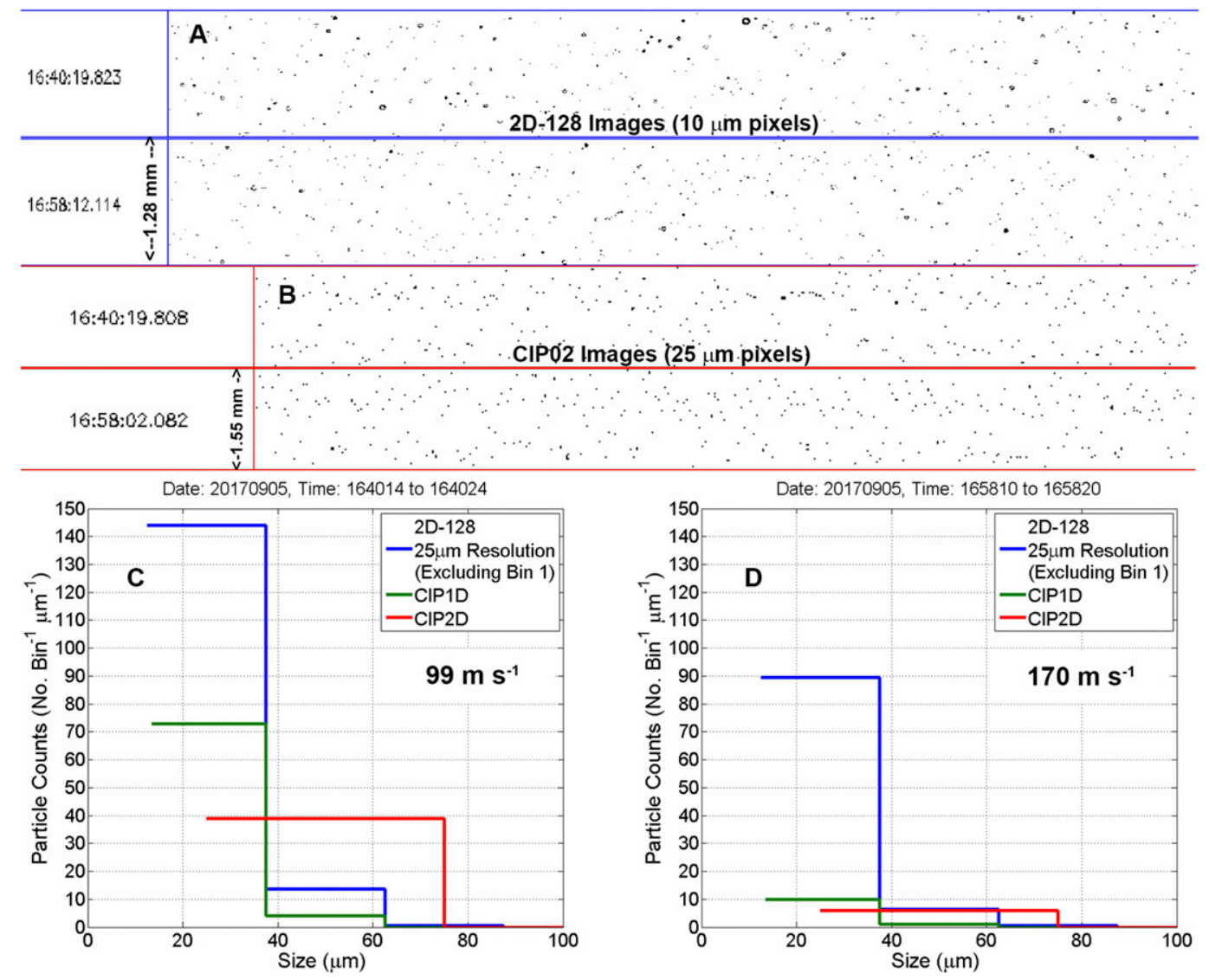

FIG. 17. (a) 2D-128 images recorded at Learjet true airspeed of (top) 99 and (bottom) $170 \mathrm{~m} \mathrm{~s}^{-1}$. (b) CIP02 images recorded at Learjet true airspeed of (top) 99 and (bottom) $170 \mathrm{~m} \mathrm{~s}^{-1}$. Particle size distributions with 2D-128 interpolated to $25-\mu \mathrm{m}$ size bins shown on linear axes at (c) 99 and (d) $170 \mathrm{~m} \mathrm{~s}^{-1}$.

Corrections to sample volume using CIP02 can be estimated using Eq. (2), which ratios the number of images recorded by an independent counter that registers a count whenever one or more photodiodes is occulted, regardless of whether the probe is in overload. Approximations to sample volume can be estimated by dividing the measured sample volume by the ratio of the number of images in the $2 \mathrm{D}$ buffer to the number of images recorded by the counter. However, this adjustment is valid only if the particle concentration is homogeneous over the measurement period, the number of images is large, and there is not particle shattering or noise affecting the particle counter during dead time. The 2D-128 can also experience dead time if the particle rate is high enough. However, the time the 2D-128 (i.e., 2D-S) goes into and comes out of overload is measured to within $50 \mathrm{~ns}$ (as opposed to measuring the number of missed images), so the sample volume can be adjusted and applied precisely to the sample of imaged particles. Thus, the only limitation is that the sum of the sampled volumes must be representative of the region of interest.
A comparison of the number of the small surface defects as the rigid acrylic disk speed was increased provided an excellent evaluation of how time response influenced the performance of the probes (Table 2). Tests with the rigid disk revealed that the number of surface defects between 10 and $100 \mu \mathrm{m}$ that were imaged by the CIPs dropped precipitously with increasing disk speed. At $176 \mathrm{~m} \mathrm{~s}^{-1}$ the number of images counted by CIP02 using 2D SODA software was $65 \%$ of the number counted at $44 \mathrm{~m} \mathrm{~s}^{-1}$. The corresponding drop in the number of 1D CIP02 images was $52 \%$. At $176 \mathrm{~m} \mathrm{~s}^{-1}$ the number of images counted by CIP17 using 2D SODA software was $50 \%$ of the number counted at $44 \mathrm{~m} \mathrm{~s}^{-1}$. The corresponding drop in the number of 1D CIP17 images was 55\%. The 2D-128 did not experience any significant degradation with increasing disk speed, recording $100 \%$ of the images at $176 \mathrm{~m} \mathrm{~s}^{-1}$ that it recorded at $44 \mathrm{~m} \mathrm{~s}^{-1}$ (Table 2).

Both a CIP02 and a 2D-S probe were installed on the SPEC Learjet to see whether the results from the rigid spinning disk could be substantiated with flight tests. 
The probes were flown in a warm stratus cloud on 5 September 2017 that also contained large ash particles from pervasive wildfires in Colorado, Montana, and California. Based on 2D-S measurements, two 10-s regions of cloud with about the same average particle concentration $\left(\sim 20 \mathrm{~cm}^{-3}\right)$ were penetrated at 99 and $170 \mathrm{~m} \mathrm{~s}^{-1}$. The 2D-128 measurements showed that the particles ranged from 10 to $80 \mu \mathrm{m}$, similar to the size range of the surface defects on the rigid disk (see Figs. 12-15 and 17). However, there was a higher percentage of $25-\mu \mathrm{m}$ particles measured at the higher speed. An analysis of the results confirmed the tendency seen in the spinning disk data but to a more exaggerated extent. A comparison at 99 and $170 \mathrm{~m} \mathrm{~s}^{-1}$ from the Learjet data showed that only $9 \%$ of the CIP02 2D particles counted at $99 \mathrm{~m} \mathrm{~s}^{-1}$ were observed at $170 \mathrm{~m} \mathrm{~s}^{-1}$. In comparison, the $2 \mathrm{D}-128$ recorded $86 \%$ of the particles counted at $170 \mathrm{~m} \mathrm{~s}^{-1}$ that were observed at $99 \mathrm{~m} \mathrm{~s}^{-1}$.

\section{Conclusions}

As stated in the introduction, the overarching purpose of this paper is to use laboratory and flight-test results from imaging probe performance to produce quantitative results that investigators can use to evaluate archived data and to better plan how measurement uncertainties will affect future data collection. Based on these results, we characterize data generated by the 2D-S (analogous to the single-channel 2D-128) and CIP in the following bullet points:

- Based on the results from this research, 2D-S particle data $\geq 10 \mu \mathrm{m}$ in size (drop diameter or particle maximum dimension), which are collected at airspeeds up to $190 \mathrm{~m} \mathrm{~s}^{-1}$ (and possibly as high as $230 \mathrm{~m} \mathrm{~s}^{-1}$ based on previous results from Lawson et al. 2006), are not degraded by the time response of the probe electronics.

- CIP02 and CIP17 data collected at particle speeds typical of research aircraft $\left(>\sim 90 \mathrm{~m} \mathrm{~s}^{-1}\right)$ are significantly affected by increasing particle speed. The effect is that the percentage of imaged particles $\leq \sim 75 \mu \mathrm{m}$ in size decrease monotonically with increasing particle speed (this effect may extend to larger particle sizes, but insufficient experimental data were available to determine whether this is the case). Based on data collected with the rigid spinning disk, the decrease in counting efficiency when the disk speed is increased from 38 to $176 \mathrm{~m} \mathrm{~s}^{-1}$ is about $50 \%$.

- As a result of a slow time response, digitization, and DOF effects creating under sizing or disappearance of one- to three-pixel particles with increasing sampling velocity, CIP uncertainties in counting and sizing particles $<\sim 75 \mu \mathrm{m}$, and 2D-S uncertainties in counting and sizing particles $<\sim 30 \mu \mathrm{m}$ can be problematic. This is especially valid in cirrus clouds, where aircraft true airspeed is typically high, the concentration of small ice particles continues to be a controversy (e.g., Jensen et al. 2009), and scattering probes exhibit intrinsic instrument uncertainties in this size range (Gardiner and Hallett 1985; Korolev et al. 2013).

- CIPs do not generate an overload flag when the electronics cannot record any more images. Adjustment to the sample volume is estimated by counting the number of missed images as reported by the CIP 16-bit particle counter. The adjustment assumes that particle concentration was homogeneous, that particle concentrations are sufficiently large to provide good counting statistics over the region of interest, and there was no particle shattering or noise generated during the dead time.

- The CIP manufactured in 2017 suffers considerably more dead time than the CIP manufactured in 2002. Laboratory tests also show that the newer CIP has an increased sample volume as a result of a larger DOF. Users are advised to contact the manufacturer to determine which version of CIP electronics and optics are in their probe.

- Dead time generated by both CIP and 2D-S electronics will have an effect on the sampling statistics of the largest particles, which are found in much smaller concentrations: the higher the percentage of dead time, the lower the probability of imaging large particles that exist in low concentrations. Using an example of a monodisperse population of $100-\mu \mathrm{m}$ diameter drops, it can be shown that CIP02 will start to experience dead time when the concentration of drops exceeds about $350 \mathrm{~L}^{-1}$ at a true airspeed of $150 \mathrm{~m} \mathrm{~s}^{-1}$. The 2D-128 will start to experience dead time in a drop concentration of about $1550 \mathrm{~L}^{-1}$ under the same conditions. If a $2 \mathrm{D}$ probe is dead for a large majority of the time-which can occur when sampling in tropical and mixed-phase convection, or there is an anvil cloud near the source of convective outflow (Lawson et al. 2010, 2017) — then there is an increased probability that large particles (drops and ice) will be undersampled or missed altogether. Accompanying measurements with imaging probes with coarser size resolution and larger sample volumes will ameliorate this situation.

- The research reported here does not offer quantitative corrections for the instrument errors that have been uncovered. Instead, it describes conditions under which the uncertainties can exist and provides some qualitative estimates of magnitude. Corrections to the data will require more laboratory tests with upgraded 
TABLE A1. Principal components of the OAP DOF calibration system (derived from Lance et al. 2010).

\begin{tabular}{|c|c|c|c|}
\hline Component description & Manufacturer/supplier & Model No./part No. & Specifications \\
\hline Piezoelectric actuator & MicroFab Inc. & MJ-ABP-01-030 & $30-\mu \mathrm{m}$ diameter \\
\hline Droplet generator controller & MicroFab Inc. & JetDrive III & Stage 1: +16 V $24 \mu$ s Stage 2: -16 V $48 \mu \mathrm{s}$ \\
\hline Evaporating flow-tube & Allen Scientific Glass & - & $\begin{array}{l}\text { 28-cm-long evaporation, glass section, ID }=2 \mathrm{~cm} \text {, } \\
\text { tapering to nozzle with ID }=0.5 \mathrm{~mm}\end{array}$ \\
\hline Water pump & McMaster-Carr & $8220 \mathrm{~K} 43$ & Fixed flow-rate pump \\
\hline Water manifold & Cole-Parmer & A-06464-85 & Four three-way valves \\
\hline Laboratory CPI camera & Balser & ACA1300-200 $\mu \mathrm{m}$ & USB 3.0 camera \\
\hline Laboratory CPI lens & Edmund Optics & Compact TL & $8 \mathrm{X}, 65-\mathrm{mm} \mathrm{WD}$ \\
\hline Laboratory CPI pulsed laser & Laser Components Inc. & L-Cube & $905-\mathrm{nm} 30 \mathrm{~nJ}$ \\
\hline Linear stages (2) & Thorlabs & $151-411 \mathrm{ME}$ & $\begin{array}{l}50-\mathrm{mm} \text { travel micrometer drives } \\
10-\mu \mathrm{m} \text { graduation }\end{array}$ \\
\hline Flow controller & Alicat Scientific & & Flow rate \\
\hline
\end{tabular}

facilities, more flight tests, and improved forward models of the instruments and numerical simulations.

Acknowledgments. This work was conducted under independent research and development funding. We appreciate the contributions of Alexei Korolev for lending CIP02, Matt Freer for supplying helpful information concerning DMT CIPs, and Aaron Bansemer for supplying SODA software and helpful information for running the code. We are very appreciative of the insightful comments offered by referees Dr. Korolev and Dr. Baumgardner, and an anonymous referee.

\section{APPENDIX}

\section{DOF Calibration Technique}

The depth of field (DOF) calibration technique for optical array probes (OAPs) is well established in the literature (Korolev et al. 1991). The technique involves moving a monodispersed stream of droplets along the $z$ axis of the OAP laser beam (perpendicular to the arms of the instrument). This is equivalent to a sample area map for a scattering probe in a single dimension. The apparatus and technique used for this study are described in detail by Lance et al. (2010), and Table A1 lists all of the equipment used for these tests. The actual size of a water droplet is determined using a high-speed pulsed laser and a long working distance (WD) $8 \mathrm{X} \mathrm{mi-}$ croscope with $0.6-\mu \mathrm{m}$ resolution to capture 8-bit images of the droplets as they pass though the OAP sample volume. Drop-size accuracy is determined by comparing measurements of glass beads with known diameters and is determined to be $\pm 1 \mu \mathrm{m}$.

Figure A1 shows an example of the raw data collected during the DOF study of a $2 \mathrm{D}-\mathrm{S}$ probe for a single droplet size. Water droplets are injected at a fixed rate of $100 \mathrm{~Hz}$ (for this test), and each position is sampled for $30 \mathrm{~s}$ (or more) to allow for reasonable sampling statics from a slight movement of the droplets as a result of fluctuations in air currents. For the example in Figure A1, the water droplet was observed to have a diameter of $37 \mu \mathrm{m}$ throughout the test. The droplet injector is positioned directly above the 2D-S laser and translated over a $10-\mathrm{mm}$ range in $0.5-\mathrm{mm}$ steps, centered on the middle of the 2D-S sample volume. As the injector moves closer to the middle of the sample volume $(Z \sim 5 \mathrm{~mm})$, the 2D-S reports 100 particles a second; however, at the edge of the DOF, the rate drops to merely a few counts per second. This rapid decay in counting efficiency is a result of the out-of-focus "doughnut" images becoming larger and then diffuse, as shown in the example in Fig. A1. Algorithms to resize out-of-focus particles are well established (Korolev 2007), but for this technique we merely consider particle count rates and not particle size.

The depth of field for a given particle size is typically defined as a length scale over which particles may

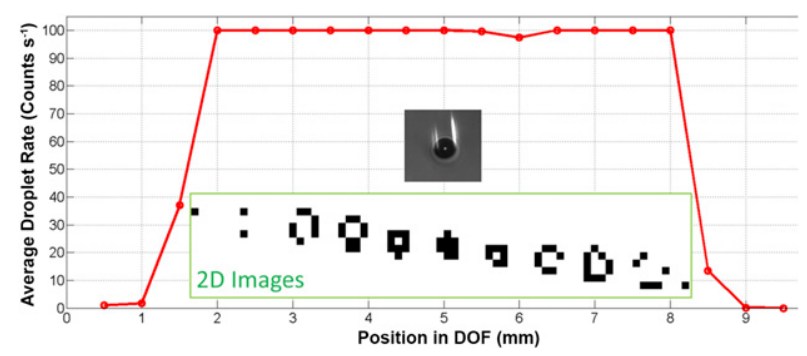

FIG. A1. Results from a DOF determination for a single channel of the 2D-S. A monodispersed stream of droplets was generated at a rate of 100 counts per second and moved along the 2D-S laser axis perpendicular to the arms $(z$ axis). The particle stream was stationary for $30 \mathrm{~s}$ at each $\Delta Z=0.5 \mathrm{~mm}$ position. Examples of how droplet images vary are shown corresponding to their position in the DOF. 

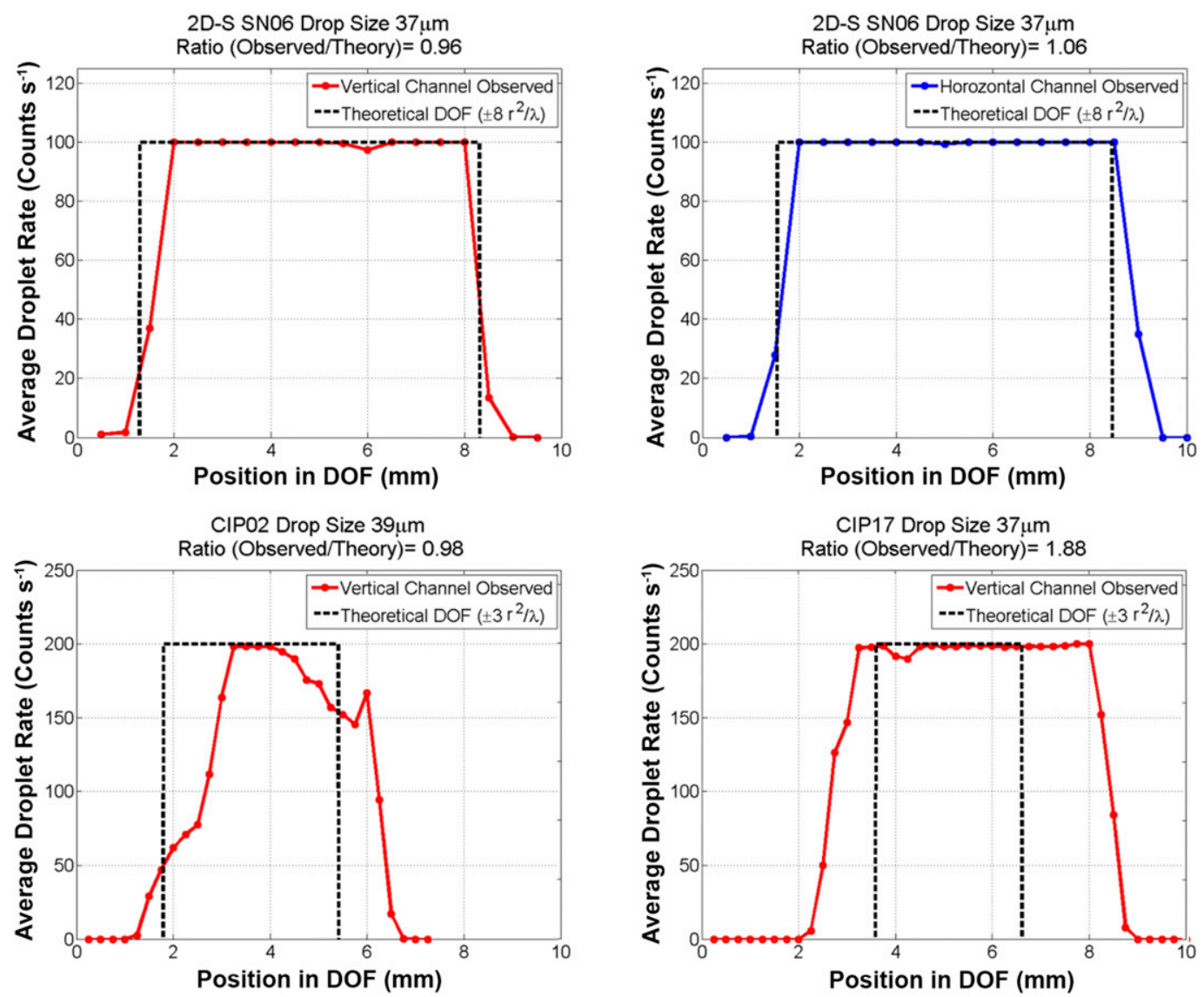

FIG. A2. DOF laboratory results for a 2D-S: (top left) vertical channel, (top right) horizontal channel, (bottom left) CIP02, and (bottom right) CIP17 (bottom right). Tests were conducted with a drop generator and the procedure described in the appendix.

be resolved by an OAP. Determining this distance is complicated by the "edge" region of the DOF where images are observed to fragment. To account for these regions, the integrated area under the average rate plot is compared to that of an idealized DOF profile (sharp edge flat top) using the probe manufacturer's specified DOF equation. In Fig. A2 the measured results (solid lines) from a 2D-S (top), the CIP02 (bottom left), and the CIP17 (bottom right) are compared to the manufacturers' idealized DOF profiles (dashed lines). For each probe a ratio between the measured and idealized integrated areas is listed. Both channels of the 2D-S fall within $10 \%$ of the manufacturer's specification. The measured DOF pattern for the CIP02 does not follow the idealized DOF pattern, but the average agreement in width of the DOF is good. The measured CIP17 DOF is sharper than for CIP02 and about twice the idealized value specified by the manufacturer. This suggests that a more thorough study of the DOF of modern CIPs is warranted (and was not possible in this work because of the unavailability of more than one modern CIP).

\section{REFERENCES}

Baumgardner, D., and A. Korolev, 1997: Airspeed corrections for optical array probe sample volumes. J. Atmos. Oceanic Technol., 14, 1224-1229, https://doi.org/10.1175/1520-0426(1997)014<1224: ACFOAP $>2.0 . C O ; 2$.

$\longrightarrow$, H. Jonsson, W. Dawson, D. O'Connor, and R. Newton, 2001: The cloud, aerosol and precipitation spectrometer (CAPS): A new instrument for cloud investigations. Atmos. Res., 59-60, 251-264, https://doi.org/10.1016/S0169-8095(01)00119-3.

Gardiner, B. A., and J. Hallett, 1985: Degradation of in-cloud forward scattering spectrometer probe measurements in the presence of ice particles. J. Atmos. Oceanic Technol., 2 , 171-180, https://doi.org/10.1175/1520-0426(1985)002<0171: DOICFS $>2.0 . \mathrm{CO} ; 2$.

Hayman, M., K. J. McMenamin, and J. B. Jensen, 2016: Response time characteristics of the Fast-2D optical array probe detector board. J. Atmos. Oceanic Technol., 33, 2569-2583, https://doi.org/10.1175/JTECH-D-16-0062.1.

Heymsfield, A. J., and J. L. Parrish, 1986: An interactive system for processing PMS imaging probe data. J. Atmos. Oceanic Technol., 3, 734-736, https://doi.org/10.1175/1520-0426(1986)003<0734: AISFPP $>2.0 . C O ; 2$.

Hobbs, P. V., and A. L. Rangno, 1998: Microstructures of low and middle-level clouds over the Beaufort Sea. Quart. 
J. Roy. Meteor. Soc., 124, 2035-2071, https://doi.org/10.1002/ qj. 49712455012.

Jensen, E. J., and Coauthors, 2009: On the importance of small ice crystals in tropical anvil cirrus. Atmos. Chem. Phys., 9, 5519-5537, https://doi.org/10.5194/acp-9-5519-2009.

— , and Coauthors, 2016: On the susceptibility of cold tropical cirrus to ice nuclei abundance. J. Atmos. Sci., 73, 2445-2464, https://doi.org/10.1175/JAS-D-15-0274.1.

Jensen, J. B., and H. Granek, 2002: Optoelectronic simulation of the PMS $260 \mathrm{X}$ optical array probe and application to drizzle in a marine stratocumulus. J. Atmos. Oceanic Technol., 19, 568-585, https://doi.org/10.1175/1520-0426(2002)019<0568: OSOTPO $>2.0 . \mathrm{CO} ; 2$.

Knollenberg, R. G., 1970: The optical array: An alternative to scattering or extinction for airborne particle size determination. J. Appl. Meteor., 9, 86-103, https://doi.org/10.1175/ 1520-0450(1970)009<0086:TOAAAT > 2.0.CO;2.

Korolev, A. V., 2007: Reconstruction of the sizes of spherical particles from their shadow images. Part I: Theoretical considerations. J. Atmos. Oceanic Technol., 24, 376-389, https:// doi.org/10.1175/JTECH1980.1.

— S. V. Kuznetsov, Y. E. Makarov, and V. S. Novikov, 1991: Evaluation of measurements of particle size and sample area from optical array probes. J. Atmos. Oceanic Technol., 8, 514-522, https://doi.org/10.1175/1520-0426(1991)008<0514: EOMOPS $>2.0 . \mathrm{CO} ; 2$.

_ J. W. Strapp, and G. A. Isaac, 1998: Evaluation of the accuracy of PMS optical array probes. J. Atmos. Oceanic Technol., 15, 708-720, https://doi.org/10.1175/1520-0426(1998)015<0708: EOTAOP $>2.0 . \mathrm{CO} ; 2$.

— E. F. Emery, J. W. Strapp, S. G. Cober, and G. A. Isaac, 2013: Quantification of the effects of shattering on airborne ice particle measurements. J. Atmos. Oceanic Technol., 30, 2527 2553, https://doi.org/10.1175/JTECH-D-13-00115.1.

Lance, S., C. A. Brock, D. Rogers, and J. A. Gordon, 2010: Water droplet calibration of a cloud droplet probe and in-flight performance in liquid, ice and mixed-phase clouds during ARCPAC. Atmos. Meas. Tech., 3, 1683-1706, https://doi.org/ 10.5194/amt-3-1683-2010.

Lawson, R. P., 2011: Effects of ice particles shattering on the 2D-S probe. Atmos. Meas. Tech., 4, 1361-1381, https://doi.org/ 10.5194/amt-4-1361-2011.

_- and P. Zuidema, 2009: Aircraft microphysical and surfacebased radar observations of summertime Arctic clouds. J. Atmos. Sci., 66, 3505-3529, https://doi.org/10.1175/ 2009JAS3177.1.

— D. O'Connor, P. Zmarzly, K. Weaver, B. A. Baker, Q. Mo, and H. Jonsson, 2006: The 2D-S (stereo) probe: Design and preliminary tests of a new airborne, high-speed, high-resolution particle imaging probe. J. Atmos. Oceanic Technol., 23, 1462-1477, https://doi.org/10.1175/JTECH1927.1.

— E. Jensen, D. L. Mitchell, B. Baker, Q. Mo, and B. Pilson, 2010: Microphysical and radiative properties of tropical clouds investigated in TC4 and NAMMA. J. Geophys. Res., 115, D00J08, https://doi.org/10.1029/2009JD013017.

— , S. Woods, and H. Morrison, 2015: The microphysics of ice and precipitation development in tropical cumulus clouds. J. Atmos. Sci., 72, 2429-2445, https://doi.org/10.1175/JAS-D14-0274.1.

—_, C. Gurganus, S. Woods, and R. Bruintjes, 2017: Aircraft observations of cumulus microphysics ranging from the tropics to midlatitudes: Implications for "new" secondary ice process. J. Atmos. Sci., 74, 2899-2920, https://doi.org/10.1175/JAS-D17-0033.1.

Mace, G. G., and S. Benson, 2017: Diagnosing cloud microphysical process information from remote sensing measurements: A feasibility study using aircraft data. Part I: Tropical anvils measured during TC4. J. Appl. Meteor. Climatol., 56, 633-649, https://doi.org/10.1175/JAMC-D-16-0083.1.

McFarquhar, G. M., and Coauthors, 2017: Processing of ice cloud in situ data collected by bulk water, scattering, and imaging probes: Fundamentals, uncertainties, and efforts toward consistency. Ice Formation and Evolution in Clouds and Precipitation: Measurement and Modeling Challenges, Meteor. Monogr., No. 58, Amer. Meteor. Soc., https://doi.org/10.1175/ AMSMONOGRAPHS-D-16-0007.1.

Mitchell, D. L., R. P. d'Entremont, and R. P. Lawson, 2010: Inferring cirrus size distributions through satellite remote sensing and microphysical databases. J. Atmos. Sci., 67, 1106-1125, https://doi.org/10.1175/2009JAS3150.1.

Pruppacher, H. R., and J. D. Klett, 1997: Microphysics of Clouds and Precipitation. Kluwer Academic Publishers, 954 pp.

Solomon, S., K. Rosenlof, R. Portmann, J. Daniel, S. Davis, T. Sanford, and G.-K. Plattner, 2010: Contributions of stratospheric water vapor changes to decadal variations in the rate of global warming. Science, 327, 1219-1223, https:// doi.org/10.1126/science.1182488.

Strapp, J. W., F. Albers, A. Reuter, A. V. Korolev, U. Maixner, E. Rashke, and Z. Vukovic, 2001: Laboratory measurements of the response of a PMS OAP-2DC. J. Atmos. Oceanic Technol., 18, 1150-1170, https://doi.org/10.1175/1520-0426 (2001)018<1150:LMOTRO > 2.0.CO;2.

Wu, W., and G. M. McFarquhar, 2016: On the impacts of different definitions of maximum dimension for nonspherical particles recorded by $2 \mathrm{D}$ imaging probes. J. Atmos. Oceanic Technol., 33, 1057-1072, https://doi.org/10.1175/JTECH-D-15-0177.1. 